\title{
A CERTAIN PROPERTY OF GEODESICS OF THE FAMILY OF RIEMANNIAN MANIFOLDS $O_{n}^{2}$ (VIII)
}

\author{
By Tominosuke OTsuki
}

\section{$\S 0$. Introduction.}

This is exactly a continuation of Part (VII) ([18]) with the same title written by the present author which proved the following conjecture is true for $5 \leqq n \leqq 9.7$. We shall show that this conjecture is also true for $4.5 \leqq n \leqq 5$ in the present paper by the same method as in Part (VII) which is a little revised one and may be valid also for $3 \leqq n \leqq 4.5$ (see the final remark of this paper), but perhaps useless for $2 \leqq n \leqq 3$, because the constant $b_{n}$, having carried out an important role in the proof of the conjecture, is defined for $2.5 \leqq n<\infty$ and monotone decreasing in $3 \leqq n<\infty$. As the previous ones we shall use the numerical data obtained by means of computors in the verification. We shall also use the same notation in the previous ones Part (I ) (VII).

The period $T$ of any non-trivial solution $x(t)$ of the non-linear differential equation of order 2 :

$$
n x\left(1-x^{2}\right) \frac{d^{2} x}{d t^{2}}+\left(\frac{d x}{d t}\right)^{2}+\left(1-x^{2}\right)\left(n x^{2}-1\right)=0
$$

with a constant $n>1$ such that $x^{2}+x^{\prime 2}<1$ is given by the integral:

$$
T=\sqrt{n c} \int_{x_{1}}^{x_{2}} \frac{d x}{x \sqrt{(n-x)\left\{x(n-x)^{n-1}-c\right\}}},
$$

where $x_{0}=n\{\min x(t)\}^{2}, x_{1}=n\{\max x(t)\}^{2}, \quad 0<x_{0}<1<x_{1}<n$ and $c=x_{0}\left(n-x_{0}\right)^{n-1}$ $=x_{1}\left(n-x_{1}\right)^{n-1}$.

CONJECTURE C. The period $T$ as a function of $\tau=\left(x_{1}-1\right) /(n-1)$ and $n$ is monotone decreasing with respect to $n(>2)$ for any fixed $\tau(0<\tau<1)$.

Here the author thanks heartily Professor Naoto Abe for his cooperation in the numerical computations by computors.

\section{$\S 1$. Preliminaries.}

Setting $T=\Omega(\tau, n)$, we have the formulas

Received January 20, 1986 


$$
\frac{\partial \Omega(\tau, n)}{\partial n}=-\frac{\sqrt{c}}{2 b^{2} n \sqrt{n}} \int_{x_{0}}^{x_{1}} \frac{(1-x) \sqrt{x(n-x)^{n-1}-c}}{x^{2}(n-x)^{n}} V\left(x, x_{1}\right) d x
$$

((7.4) and Proposition 3 in (III)), where $b=\sqrt{B-c}, B=(n-1)^{n-1}$ and $V\left(x, x_{1}\right)$ are defined as follows:

$$
V\left(x, x_{1}\right):=\frac{x^{2} N\left(x, x_{1}\right)}{(1-x)^{5} \sqrt{n-x}}+\frac{X^{2} N\left(X, x_{1}\right)}{(X-1)^{5} \sqrt{n-X}},
$$

where

$$
\begin{aligned}
& f_{0}(z):=(2 n-1-z) B-(n-z)^{n-1}\left\{n-z+(n-1) z^{2}\right\}, \\
& f_{1}(z):=\{4 n-1-(2 n+1) z\} B-(n-z)^{n-1}\left\{n+(2 n-1) z-(n+1) z^{2}\right\}, \\
& F_{2}(z):=-P_{2}(z) B+(n-z)^{n-1} P_{3}(z), \\
& P_{2}(z):=(2 n+1) z^{2}-2\left(2 n^{2}+5 n-4\right) z+16 n^{2}-16 n+3, \\
& P_{3}(z):=-(n-1) z^{3}+\left(2 n^{2}-7 n+8\right) z^{2}+(n-3)(4 n-1) z+3 n(2 n-1), \\
& \lambda(z):=\log (n-z)+\frac{n-1}{n-z}, \quad \psi(z):=z(n-z)^{n-1}, \\
& \tilde{\lambda}(z):=\lambda(z)-\frac{n}{n-1} \frac{(z-1)^{2}}{z(n-z)}=\log (n-z)+\frac{n z-1}{(n-1) z}, \\
& N\left(z, x_{1}\right):=(n-z) F_{2}(z)\left\{\lambda(z)-\tilde{\lambda}\left(x_{1}\right)\right\} \\
& \quad \quad+3(z-1)^{2} f_{0}(z)-2 n(z-1)^{3}\left\{B-z(n-z)^{n-1}\right\},
\end{aligned}
$$

and $X=X_{n}(x), 0<x<1<X<n$, defined by $\psi(x)=\psi(X)$.

$V\left(x, x_{1}\right)$ is increasing with respect to $x_{1}$ in $X_{n}(x) \leqq x_{1}<n$ for $0<x<1$ and $V\left(x, X_{n}(x)\right)$ is negative near $x=0$ when $2<n<\frac{5+\sqrt{13}}{4}=2.15138 \cdots$ and near $x=1$ when $2<n<\frac{1+\sqrt{13}}{2}=2.30277 \cdots$, by Lemma 8.1 in (III). We shall show that $V\left(x, X_{n}(x)\right)>0$ when $3 \leqq n \leqq 5$, which implies $\partial \Omega(\tau, n) / \partial n<0$ by $(1.1)$. We have the formula (8.1) in (III):

$$
V(x, X(x))=U_{0}(x)-U_{1}(x)+U_{2}(x)+U_{4}(x)+U_{5}(x)-U_{6}(x),
$$

where $X(x)=X_{n}(x)$ and

$$
\begin{aligned}
& U_{0}(x):=\frac{x^{2} \sqrt{n-x}}{(1-x)^{5}} F_{2}(x)\{\lambda(x)-\tilde{\lambda}(X(x))\}, \quad U_{1}(x):=\frac{3 x^{2} f_{0}(x)}{(x-1)^{3} \sqrt{n-x}}, \\
& U_{2}(x):=\frac{2 n x^{2}\{B-\psi(x)\}}{(1-x)^{2} \sqrt{n-x}}, \quad U_{3}(x):=\frac{n x F_{2}(x)}{(n-1)(x-1)^{3} \sqrt{n-x}}, \\
& U_{4}(x):=U_{3}(X(x)), \quad U_{5}(x):=U_{1}(X(x)), \quad U_{6}(x):=U_{2}(X(x)),
\end{aligned}
$$


and the inequality (2.4) in (VI):

$$
\begin{aligned}
V(x, X(x))> & \frac{2 n(n-x)^{2(n-1)}\{B-\phi(x)\} \sqrt{B}}{\left\{B-(n-x)^{n-1}\right\}^{2} \sqrt{n B-(n-x)^{n-1}}}+\frac{2 n X \sqrt{n-X} B}{(n-1)(X-1)^{3}} H(X) \\
& +\frac{x^{2} \sqrt{n-x} F_{2}(x)\{\lambda(x)-\tilde{\lambda}(X)\}}{(1-x)^{5}} \quad \text { for } 0<x<1,
\end{aligned}
$$

where

$$
H(X):=\left(\frac{n-X}{n-1}\right)^{n-1}\left\{(n-1) X^{2}+(2 n-5) X+3 n\right\}-\{8 n-5-(2 n+1) X\} .
$$

All the factors except $H(X)$ in the right hand side of (1.13) are positive. For $n \geqq 2.5$, we defined the constant $b_{n}$ by $H(b)=0,1<b \leqq n$. Regarding $b_{n}$, we have the following

LEMMA 1.1. $b_{n}$ has the following properties:

i ) $b_{n}>\frac{2 n+10}{2 n+1}$ for $n>2.5$;

ii) $b_{2.5}=b_{3}=2.5,2.5<b_{n}<n$ for $2.5<n<3,2.25<b_{n}<2.5$ for $3<n<4$ and $b_{n}<2.268$ for $n \geqq 4$;

iii) $b_{n}$ is decreasing in $3<n<\infty$.

(See Lemma 2.2, Lemma 2.3, Lemma 2.4 and Theorem 1 in [20]).

Since we have

$$
H(X) \geqq 0 \text { for } b_{n} \leqq X<n \text { and } H(X)<0 \text { for } 1<X<b_{n},
$$

it is sufficient to prove $V\left(x, X_{n}(x)\right)>0$ for $X_{n}^{-1}\left(b_{n}\right)<x<1$ for our purpose.

Regarding the evaluation of $X_{n}^{-1}(X)$, we cite some results. We set

$$
w=w(n, X):=\left(\frac{n-X}{n-1}\right)^{n-1} \quad \text { for } 1<X<n .
$$

LEMMA 1.2. $w(n, X)$ is increasing with respect to $n(>1)$ with $1<X<n$ and decreasing with respect to $X$ in $1<X<n$. When $n>2$, we have

$$
w(n, X)<x=X_{n}^{-1}(X) \quad \text { for } \quad 1<X<n .
$$

(Lemma 4.1 in (VII)).

We set for $n \geqq 2,1<C<n$,

$$
k_{n}(C):=\frac{n+1+(n-1) C-\sqrt{(n-1)\left\{(n-1) C^{2}-2(n+1) C+5 n-1\right\}}}{2 C},
$$

and obtain

$$
n-k_{n}(C)=\frac{(n+1)(C-1)+\sqrt{(n-1)\left\{(n-1) C^{2}-2(n+1) C+5 n-1\right\}}}{2 C} .
$$


LEMMA 1.3. i) $k_{n}(C)$ is increasing with respect to $C$ in $1<C \leqq \frac{5 n-1}{2(n+1)}$, with $n>2$, and

$$
k_{n}(1)=1 \quad \text { and } \quad k_{n}\left(\frac{5 n-1}{2(n+1)}\right)=\frac{(n+1)^{2}}{5 n-1} .
$$

ii) $k_{n}(C)$ is increasing with respect to $n(\geqq 2.5)$ and $n-k_{n}(C)$ is increasing with respect to $n(>2)$, with $1<C \leqq \frac{5 n-1}{2(n+1)}$.

Proof. i) and the first part of ii) are proved in Lemma 2.3 in (VII). Regarding the second part, it is evident from

$$
\begin{aligned}
& \frac{\partial}{\partial n}\left(n-k_{n}(C)\right) \\
= & \frac{(n-1) C^{2}-2 n C+5 n-3+(C-1) \sqrt{(n-1)\left\{(n-1) C^{2}-2(n+1) C+5 n-1\right\}}}{2 C \sqrt{(n-1)\left\{(n-1) C^{2}-2(n+1) C+5 n-1\right\}}}
\end{aligned}
$$

and $(n-1) C^{2}-2 n C+5 n-3>0$ and $(n-1) C^{2}-2(n+1) C+5 n-1>0$ for any $C$ and $n>2$.

Q.E.D.

LEMMA 1.4. When $n>2$, for $1<C \leqq \frac{5 n-1}{2(n+1)}, k_{n}(C) \leqq k<n-1$ and $1<X \leqq C$ we have

$$
x=X_{n}^{-1}(X)<\left(\frac{n-X}{n-1}\right)^{n-k} .
$$

(Lemma 2.2 in (VII)).

Noticing

$$
n-k_{n}\left(\frac{5 n-1}{2(n+1)}\right)=\frac{(n-1)(4 n+1)}{5 n-1}
$$

we set

$$
y=y(n, X):=\left(\frac{n-X}{n-1}\right)^{\frac{(n-1)(4 n+1)}{5 n-1}} \text { for } 1<X<n \text {. }
$$

LEMMA 1.5. $y(n, X)$ is increasing with respect to $n(>1)$, with $1<X<n$, and decreasing with respect to $X$ in $1<X<n$, with $n>1$.

(Lemma 4.3 in (VII)).

§2. Evaluation of $V\left(x, X_{n}(x)\right)$ near $x=1$ for $3 \leqq n<5$.

We shall make an analogous formula on the evaluation of $V\left(x, X_{n}(x)\right)$ near $x=1$ for $3 \leqq n \leqq 5$ to those in Proposition 4 in $\S 5$ of (VII).

First, we shall investigate the case of $3 \leqq n<4$.

LEMMA 2.1. We have 


$$
\begin{aligned}
& \frac{U_{o}(x)}{\sqrt{n-1}}> \frac{n^{2}\left(n^{2}-n+1\right)}{6}(n-1)^{n-4}(X-1) \\
&-\frac{n\left(n^{2}-n+1\right)\left\{3 n(2 n-13) p_{n}+18 n^{2}-28 n-4\right\}}{108}(n-1)^{n-5}(X-1)^{2} \\
&+\frac{n\left(n^{2}-n+1\right)(4 n-5) p_{n}\left(6 n p_{n}+18 n^{2}-28_{n}-4\right)}{216}(n-1)^{n-6}(X-1)^{3} \\
& \text { for } \quad 1<X \leqq C \leqq 3 / 2,3 \leqq n \leqq 4,
\end{aligned}
$$

where

$$
p_{n}=\frac{(n-1)^{n-k}}{(n-k)(n-C)^{n-k-1}}, \quad k=k_{n}(C)
$$

and

$$
\frac{X-1}{1-x}<p_{n}
$$

Proof. By using partially the argument in the proof of Lemma 5.1 in (VII) and assuming $3 \leqq n \leqq 4$, we have

$$
\begin{aligned}
& \frac{d}{d X} \frac{(2-X)(n-X)^{n-3}}{(n-x)^{n}}=-\frac{(n-2)(3-X)}{(n-x)^{n}(n-X)^{4-n}}-\frac{n(X-1)(2-X)(n-X)^{2 n-5}}{(1-x)(n-x)^{2 n-1}} \\
&>-\frac{(n-2) \cdot 2}{(n-1)^{n}(n-C)^{4-n}}-\frac{n p_{n}}{(n-1)^{4}} \\
&=-\frac{1}{(n-1)^{4}}\left\{2(n-2)\left(\frac{n-1}{n-C}\right)^{4-n}+n p_{n}\right\} \\
& \text { for } \quad 1<X \leqq C \leqq 3 / 2,
\end{aligned}
$$

since we have

$$
\frac{3}{2}<\frac{5 n-1}{2(n+1)}
$$

and (2.3) holds by Lemma 2.5 in (VII). On the other hand we have

$$
\left(\frac{n-1}{n-C}\right)^{4-n} \leqq\left(\frac{n-1}{n-1.5}\right)^{4-n} \leqq\left(\frac{4}{3}\right)^{4-n} \leqq \frac{4}{3},
$$

and hence

$$
\frac{d}{d X} \frac{(2-X)(n-X)^{n-3}}{(n-x)^{n}}>-\frac{1}{(n-1)^{4}}\left\{\frac{8(n-2)}{3}+n p_{n}\right\}
$$

and so

$$
\frac{(n-1)(2-X)(n-X)^{n-3}}{(n-x)^{n}}>\frac{1}{(n-1)^{2}}-\frac{1}{(n-1)^{3}}\left\{\frac{8(n-2)}{3}+n p_{n}\right\}(X-1) .
$$

Thus, we obtain 


$$
\begin{aligned}
& \frac{d^{2}}{d X^{2}}\{\lambda(x)-\tilde{\lambda}(X)\} \\
= & \frac{(n-1)(2-X)(n-X)^{n-3}}{(n-x)^{n}}-\frac{n(X-1)^{2}(n-X)^{2 n-4}}{(1-x)(n-x)^{2 n-1}}+\frac{2}{(n-1) X^{3}}+\frac{1}{(n-X)^{2}} \\
> & \frac{1}{(n-1)^{2}}-\frac{1}{(n-1)^{3}}\left\{\frac{8(n-2)}{3}+n p_{n}\right\}(X-1)-\frac{n p_{n}}{(n-1)^{3}}(X-1)+\frac{2}{n-1} \\
\quad & \quad-\frac{6}{n-1}(X-1)+\frac{1}{(n-1)^{2}}+\frac{2}{(n-1)^{3}}(X-1) \\
= & \frac{2 n}{(n-1)^{2}}-\frac{X-1}{3(n-1)^{3}}\left(6 n p_{n}+18 n^{2}-28 n-4\right)
\end{aligned}
$$

and hence

$$
\lambda(x)-\tilde{\lambda}(X)>\frac{n}{(n-1)^{2}}(X-1)^{2}-\frac{6 n p_{n}+18 n^{2}-28 n-4}{18(n-1)^{3}}(X-1)^{3} .
$$

Finally we obtain

$$
\begin{aligned}
U_{0}(x)= & \frac{x^{2} \sqrt{n-x}}{(1-x)^{5}} F_{2}(x)\{\lambda(x)-\tilde{\lambda}(X)\} \\
> & \frac{n^{2}\left(n^{2}-n+1\right)(n-1)^{n-3.5}}{6}\left\{1-\frac{(4 n-5) p_{n}}{2(n-1)}-(X-1)\right\} \\
& \quad \times\left\{1-\frac{6 n p_{n}+18 n^{2}-28 n-4}{18 n(n-1)^{3}}(X-1)\right\}(X-1),
\end{aligned}
$$

which can be written as the inequality of the statement of this lemma.

Q.E. D.

LEMMA 2.2. We have

where

$$
\begin{aligned}
\frac{U_{5}(x)}{\sqrt{n-1}}> & \frac{n(2 n-1)}{2}(n-1)^{n-3}+\frac{n\left(5 n^{2}-3 n+1\right)}{4}(n-1)^{n-4}(X-1) \\
& +\left\{\frac{n(n-2)(n-3) q_{n}(C)}{40(n-1)}-\frac{n(n-2)(3 n-1)(4 n-3)}{8}\right\}(n-1)^{n-5}(X-1)^{2} \\
& +\frac{n(n-2)(n-3)(4 n-3) q_{n}(C)}{80(n-1)}(n-1)^{n-6}(X-1)^{3} \\
& \text { for } 1<X \leqq C \leqq 2, \quad n \geqq 3,
\end{aligned}
$$

$$
q_{n}(C)=\left(n^{2}-1\right) C^{2}-\left(10 n^{2}-9 n-4\right) C+3 n(7 n-8) .
$$

(Lemma 5.2 in (VII)).

\section{LEMMA 2.3. We have}




$$
\begin{aligned}
&-\frac{U_{1}(x)}{\sqrt{n-1}>}>-\frac{n(2 n-1)}{2}(n-1)^{n-3}+\frac{n\left(5 n^{2}-3 n+1\right)}{4}(n-1)^{n-4}(1-x) \\
&+\frac{\mid n\left(16 n^{3}-124 n^{2}+46 n-9\right)}{80}(n-1)^{n-5}(1-x)^{2} \\
&-\frac{n(n-2)\left(24 n^{3}+224 n^{2}-221 n+39\right)}{160}(n-1)^{n-6}(1-x)^{3} \\
&-\frac{3 n(n-2)(n-3)(4 n-1)\left(8 n^{2}-8 n+3\right)}{320}(n-1)^{n-7}(1-x)^{4} \\
& \text { for } \quad 0<x<1,3 \leqq n<4 .
\end{aligned}
$$

Proof. By (5.4) in (VII) we have

$$
\begin{aligned}
f_{o}^{(6)}(x)=- & n(n-1)(n-2)(n-3)(n-4) \\
& \times\left\{\left(n^{2}-1\right) x^{2}-\left(12 n^{2}-11 n-5\right) x+n(31 n-35)\right\}(n-x)^{n-7} .
\end{aligned}
$$

Since we have

$$
\frac{12 n^{2}-11 n-5}{2\left(n^{2}-1\right)}>1 \quad \text { for } \quad n \geqq 2
$$

and

$$
\left[\left(n^{2}-1\right) x^{2}-\left(12 n^{2}-11 n-5\right) x+n(31 n-35)\right]_{x=1}=4(n-1)(5 n-1)>0,
$$

so

which implies

$$
f_{o}^{(6)}(x) \geqq 0 \quad \text { for } \quad 0 \leqq x \leqq 1, \quad \text { with } \quad 3 \leqq n<4 \text {, }
$$

$$
f_{o}^{(5)}(x) \leqq f_{o}^{(5)}(1)=3 n(n-2)(n-3)(4 n-1)(n-1)^{n-4} .
$$

Hence, by means of the argument used in the proof of Lemma 5.3 in (VII), we obtain the inequality:

$$
\begin{aligned}
f_{o}(x)> & -\frac{n(2 n-1) B}{6(n-1)}(1-x)^{3}-\frac{n(n-2)(3 n-1) B}{12(n-1)^{2}}(1-x)^{4} \\
& -\frac{n(n-2)(n-3)(4 n-1) B}{40(n-1)^{3}}(1-x)^{5} \quad \text { for } \quad 0<x<1,
\end{aligned}
$$

Using the inequality (5.9) in (VII) as follows:

$$
\frac{x^{2}}{\sqrt{n-x}}<\frac{1}{\sqrt{n-1}}-\frac{4 n-3}{2(n-1)^{1.5}}(1-x)+\frac{8 n^{2}-8 n+3}{8(n-1)^{2.5}}(1-x)^{2} \quad \text { for } \quad 0<x<1 .
$$

we obtain

$$
\begin{aligned}
-U_{1}(x)= & \frac{3 x^{2} f_{0}(x)}{(1-x)^{3} \sqrt{n-x}}>-\frac{1}{\sqrt{n-1}}\left\{1-\frac{4 n-3}{2(n-1)}(1-x)+\frac{8 n^{2}-8 n+3}{8(n-1)^{2}}(1-x)^{2}\right\} \\
& \times \frac{n B}{2(n-1)}\left\{2 n-1+\frac{(n-2)(3 n-1)}{2(n-1)}(1-x)+\frac{3(n-2)(n-3)(4 n-1)}{20(n-1)^{2}}(1-x)^{2}\right\}
\end{aligned}
$$


which can be written as (2.6), by removing the braces and arranging in order of powers of $1-x$.

Q.E.D.

LEMMA 2.4. We have

$$
\begin{aligned}
\frac{U_{2}(x)}{\sqrt{n-1}}> & n^{2}(n-1)^{n-3}-\frac{n^{2}(8 n-1)}{6}(n-1)^{n-4}(1-x) \\
& -\frac{n^{2}(n-2)(4 n-3)}{3}(n-1)^{n-5}(1-x)^{2} \text { for } 0<x<1, n>3 .
\end{aligned}
$$

(Lemma 5.4 in (VII)).

LEMMA 2.5. We have

$$
-\frac{U_{6}(x)}{\sqrt{n-1}}>-n^{2}(n-1)^{n-3}-\frac{n^{2}(8 n-1)}{6}(n-1)^{n-4}(X-1)
$$

$$
\begin{aligned}
& +\frac{n^{2}\left\{\left(20 C^{2}-115 C+88\right) n^{2}-\left(55 C^{2}-377 C+395\right) n+3\left(C^{2}-50 C+50\right)\right\}}{15(3-C)^{2}}(n-1)^{n-5}(X-1)^{2} \\
& -\frac{(4-C) n^{2}(n-2)(n-3)(4 n-3)}{6(3-C)^{2}}(n-1)^{n-6}(X-1)^{3} \quad \text { for } \quad 1<X \leqq C \leqq \frac{3}{2}, 3 \leqq n<4 .
\end{aligned}
$$

Proof. We shall use the argument of the proof of Lemma 5.5 in (VII). For $X(1<X \leqq C)$ there exists $X_{1}\left(1<X_{1}<X\right)$ such that

$$
\begin{aligned}
B-X(n-X)^{n-1}= & \frac{n(n-1)^{n-2}}{2}(X-1)^{2}-\frac{n(n-2)(n-1)^{n-3}}{3}(X-1)^{3} \\
& +\frac{n(n-1)(n-2)(n-3)\left(4-X_{1}\right)\left(n-X_{1}\right)^{n-5}}{24}(X-1)^{4} .
\end{aligned}
$$

Since we have

$$
\frac{\partial}{\partial x}(4-x)(n-x)^{n-5}=(4-n)(5-x)(n-x)^{n-6}>0 \quad \text { for } \quad 0<x<n<4,
$$

which implies

we obtain

$$
\left(4-X_{1}\right)\left(n-X_{1}\right)^{n-5}<(4-C)(n-C)^{n-5},
$$

$$
\begin{aligned}
& \frac{B-X(n-X)^{n-1}}{(X-1)^{2}}<\frac{n(n-1)^{n-2}}{2} \\
& \quad \times\left\{1-\frac{2(n-2)}{3(n-1)}(X-1)+\frac{(n-2)(n-3)(4-C)}{12(n-1)^{2}}\left(\frac{n-1}{n-C}\right)^{5-n}(X-1)^{2}\right\} .
\end{aligned}
$$

Furthermore

$$
\frac{d}{d n} \log \left(\frac{n-1}{n-C}\right)^{5-n}=-\log \frac{n-1}{n-C}-(5-n) \frac{C-1}{(n-1)(n-C)}<0 \quad \text { for } \quad 3 \leqq n<4
$$


and

$$
\left(\frac{n-1}{n-C}\right)^{5-n} \leqq\left(\frac{2}{3-C}\right)^{2}=\frac{4}{(3-C)^{2}}
$$

Hence we obtain

$$
\begin{aligned}
& \frac{B-X(n-X)^{n-1}}{(X-1)^{2}}<\frac{n(n-1)^{n-2}}{2} \\
& \quad \times\left\{1-\frac{2(n-2)}{3(n-1)}(X-1)+\frac{(n-2)(n-3)}{3(n-1)^{2}} \cdot \frac{(4-C)}{(3-C)^{2}}(X-1)^{2}\right\} \\
& \text { for } 1<X \leqq C \leqq 3 / 2,3 \leqq n<4 .
\end{aligned}
$$

Next, we have (5.14) in (VII) as

$$
\frac{X^{2}}{\sqrt{n-X}}<\frac{1}{\sqrt{n-1}}\left\{1+\frac{4 n-3}{2(n-1)}(X-1)+\frac{8 n^{2}-8 n C+3 C^{2}}{8(n-C)^{2}} \cdot \sqrt{\frac{n-1}{n-C}}(X-1)^{2}\right\} .
$$

For simplicity, taking into consideration of $3 \leqq n<4$ and $C \leqq 3 / 2$, we have

$$
\begin{aligned}
& \frac{1}{8(n-C)^{2}} \sqrt{\frac{n-1}{n-C}}=\frac{1}{8(n-1)^{2}}\left(\frac{n-1}{n-C}\right)^{5 / 2} \leqq \frac{1}{8(n-1)^{2}} \cdot \frac{4 \sqrt{2}}{(3-C)^{2} \sqrt{3-C}} \\
\leqq & \frac{\sqrt{2}}{2(n-1)^{2}(3-C)^{2} \sqrt{3-C}} \leqq \frac{1}{(n-1)^{2}(3-C)^{2} \sqrt{3}}<\frac{3}{5(n-1)^{2}(3-C)^{2}} .
\end{aligned}
$$

Hence we obtain

$$
\frac{X^{2}}{\sqrt{n-X}}<\frac{1}{\sqrt{n-1}}\left\{1+\frac{4 n-3}{2(n-1)}(X-1)+\frac{3\left(8 n^{2}-8 n C+3 C^{2}\right)}{5(n-1)^{2}(3-C)^{2}}(X-1)^{2}\right\}
$$

From (2.9) and (2.10) we obtain

$$
\text { for } 1<X \leqq C \leqq 3 / 2,3 \leqq n<4 \text {. }
$$

$$
\begin{aligned}
-U_{6}(x) & =-\frac{2 n X^{2}\left\{B-X(n-X)^{n-1}\right\}}{(X-1)^{2} \sqrt{n-X}} \\
>-n^{2}(n-1)^{n-5 / 2} & \left\{1+\frac{4 n-3}{2(n-1)}(X-1)+\frac{3\left(8 n^{2}-8 n C+3 C^{2}\right)}{5(n-1)^{2}(3-C)^{2}}(X-1)^{2}\right\} \\
& \times\left\{1-\frac{2(n-2)}{3(n-1)}(X-1)+\frac{(n-2)(n-3)(4-C)}{3(n-1)^{2}(3-C)^{2}}(X-1)^{2}\right\}
\end{aligned}
$$

and, arranging in order of powers of $X-1$,

$$
\begin{aligned}
& -\frac{U_{6}(x)}{\sqrt{n-1}}>-n^{2}(n-1)^{n-3}-\frac{n^{2}(8 n-1)}{6(n-1)}(n-1)^{n-4}(X-1) \\
& +\frac{n^{2}\left\{\left(20 C^{2}-115 C+88\right) n^{2}-\left(55 C^{2}-377 C+395\right) n+3\left(C^{2}-50 C+50\right)\right\}}{15(3-C)^{2}}(n-1)^{n-5}(X-1)^{2} \\
& -\frac{(4-C) n^{2}(n-2)(n-3)(4 n-3)}{6(3-C)^{2}}(n-1)^{n-6}(X-1)^{3}
\end{aligned}
$$




$$
\begin{aligned}
+\frac{n^{2}(n-2)\left(8 n^{2}-8 n C+3 C^{2}\right)}{5(3-C)^{4}}(n-1)^{n-7} & \\
& \times\left[2(n-1)(3-C)^{2}-(n-3)(4-C)(X-1)\right](X-1)^{3} .
\end{aligned}
$$

Regarding the last expression, we see

and

$$
8 n^{2}-8 n C+3 C^{2}=8(n-C / 2)^{2}+C^{2}>0
$$

$$
\begin{aligned}
& 2(n-1)(3-C)^{2}-(n-3)(4-C)(X-1) \\
\geqq & 2(n-1)(3-C)^{2}-(n-3)(4-C)(C-1) \\
\geqq & 2(n-1) 9 / 4-(n-3)(4-3 / 2)(3 / 2-1) \\
= & (13 n-3) / 4>0 \quad \text { for } \quad 1<X \leqq C \leqq 3 / 2,3 \leqq n<4 .
\end{aligned}
$$

Therefore, omitting this expression from the above inequality, we can obtain (2.8).

Q.E.D.

LEMMA 2.6. We have

$$
\begin{aligned}
& \frac{U_{4}(x)}{\sqrt{n-1}}> \frac{n^{2}\left(n^{2}-n+1\right)}{6}(n-1)^{n-4}(X-1) \\
&-\frac{n^{2}\left(2 n^{3}-4 n^{2}+6 n-3\right)}{12}(n-1)^{n-5}(X-1)^{2} \\
&-\frac{n^{2}(2 n-1)\left(4 n^{3}-7 n^{2}+9 n-4\right)}{24}(n-1)^{n-6}(X-1)^{3} \\
& \text { for } 1<X \leqq C \leqq 3 / 2,3 \leqq n<5 .
\end{aligned}
$$

Proof. Using the argument in the proof of Lemma 5.6 in (VII), we have

$$
F_{2}(x)>\frac{n(n-1)^{3}\left(n^{2}-n+1\right)}{6}(X-1)^{4}\left\{1-\frac{4 n^{3}-7 n^{2}+9 n-4}{2(n-1)\left(n^{2}-n+1\right)}(X-1)\right\}\left(n-X_{1}\right)^{n-5},
$$

where $X_{1}$ is a value such that $1<X_{1}<X \leqq C \leqq 3 / 2$. Since

and

$$
\left(n-X_{1}\right)^{n-5}>(n-1)^{n-5}
$$

$$
\frac{X}{\sqrt{n-X}}>\frac{1}{\sqrt{n-1}}\left\{1+\frac{2 n-1}{2(n-1)}(X-1)\right\} \quad \text { for } \quad 1<X<n
$$

Therefore, we obtain from these

$$
\begin{aligned}
U_{4}(x)=\frac{n X F_{2}(X)}{(n-1)(X-1)^{3} \sqrt{n-X}}>\frac{n^{2}\left(n^{2}-n+1\right)(n-1)^{n-3.5}}{6}(X-1) \\
\quad \times\left\{1-\frac{4 n^{3}-7 n^{2}+9 n-4}{2(n-1)\left(n^{2}-n+1\right)}(X-1)\right\}\left\{1+\frac{2 n-1}{2(n-1)}(X-1)\right\}
\end{aligned}
$$




$$
\begin{gathered}
=\frac{n^{2}\left(n^{2}-n+1\right)(n-1)^{n-3.5}}{6}(X-1)-\frac{n^{2}\left(2 n^{3}-4 n^{2}+6 n-3\right)}{12}(n-1)^{n-4.5}(X-1)^{2} \\
-\frac{n^{2}(2 n-1)\left(4 n^{3}-7 n^{2}+9 n-4\right)}{24}(n-1)^{n-5.5}(X-1)^{3} .
\end{gathered}
$$

Proposition 1. We have

Q.E. D.

$$
\begin{aligned}
& \frac{V\left(x, X_{n}(x)\right)}{n(n-1)^{n-3.5}(X-1)}>\frac{(2 n-1)\left(n^{2}-n-3\right)}{6}-\frac{X-1}{n-1} \\
& \times\left[\frac{40 n^{4}+552 n^{3}-1498 n^{2}+1152 n-153}{240}\right. \\
& \quad+\frac{\left(n^{2}-n+1\right)\left\{3 n(12 n-13) p_{n}+18 n^{2}-28 n-4\right\}}{108}-\frac{(n-2)(n-3)}{40(n-1)} q_{n}(C) \\
& \left.\quad-\frac{n\left\{\left(20 n^{2}-55 n+3\right) C^{2}-\left(115 n^{2}-377 n+150\right) C+88 n^{2}-395 n+150\right\}}{15(3-C)^{2}}\right]
\end{aligned}
$$$$
-\left(\frac{X-1}{n-1}\right)^{2}\left[\frac{160 n^{5}-288 n^{4}+1028 n^{3}-2347 n^{2}+1523 n-234}{480}\right.
$$$$
-\frac{(4 n-5)\left(n^{2}-n+1\right) p_{n}\left(6 n p_{n}+18 n^{2}-28 n-4\right)}{216}-(n-2)(n-3)(4 n-3)
$$$$
\left.\times\left\{\frac{1}{80(n-1)} q_{n}(C)-\frac{(4-C) n}{6(3-C)^{2}}\right\}\right]
$$$$
-\left(\frac{X-1}{n-1}\right)^{3} \frac{3(n-2)(n-3)(4 n-1)\left(8 n^{2}-8 n+3\right)}{320} \quad \text { for } \quad 1<X \leqq C \leqq 3 / 2,3 \leqq n<4 \text {, }
$$

where $p_{n}$ is given by (2.2) and $q_{n}(C)$ is given by (2.5).

Proof. Using Lemma 2.1-Lemma 2.6 in the expression of $V\left(x, X_{n}(x)\right)$ :

$$
\begin{aligned}
V\left(x, X_{n}(x)\right) & =U_{0}(x)-U_{1}(x)+U_{2}(x)+U_{4}(x)+U_{5}(x)-U_{6}(x) \\
& =U_{0}(x)+U_{5}(x)-U_{1}(x)+U_{2}(x)-U_{6}(x)+U_{4}(x)
\end{aligned}
$$

and noticing the facts as

the sum of the coefficients of $(n-1)^{n-4}(1-x)$ :

$$
\frac{n\left(5 n^{2}-3 n+1\right)}{4}-\frac{n^{2}(8 n-1)}{6}=-\frac{n\left(n^{2}+7 n-3\right)}{12}<0,
$$

the sum of the coefficients of $(n-1)^{n-5}(1-x)^{2}$ :

$$
\frac{n\left(16 n^{3}-124 n^{2}+46 n-9\right)}{80}-\frac{n^{2}(n-2)(4 n-3)}{3}=-\frac{n\left(272 n^{3}-508 n^{2}+342 n+27\right)}{240}<0,
$$

and the coefficients of $(n-1)^{n-6}(1-x)^{3}$ and $(n-1)^{n-7}(1-x)^{4}$ are also negative for 
$3 \leqq n<4$, and $X_{n}(x)-1>1-x$ for $0<x<1$ (by Lemma 6.2 in (IV), which says $x>2-X_{n}(x)$ for $0<x<1$ and $n>2$ ), we may replace $1-x$ by $X-1$ in these evaluating expressions. Then we compute the coefficients of $n(n-1)^{n-3-m}(X-1)^{m}$, $m=0,1,2,3,4$. The coefficient corresponding to $m=0$ vanishies ;

$$
\begin{aligned}
& n(n-1)^{n-4}(X-1): \frac{n\left(n^{2}-n+1\right)}{6}+\frac{5 n^{2}-3 n+1}{4}+\frac{5 n^{2}-3 n+1}{4} \\
& -\frac{n(8 n-1)}{6}-\frac{n(8 n-1)}{6}+\frac{n\left(n^{2}-n+1\right)}{6} \\
& =\frac{2 n^{3}-3 n^{2}-5 n+3}{6}=\frac{(2 n-1)\left(n^{2}-n-3\right)}{6} \text {; } \\
& n(n-1)^{n-5}(X-1)^{2}:-\frac{\left(n^{2}-n+1\right)\left\{3 n(12 n-13) p_{n}+18 n^{2}-18 n-4\right\}}{108}+(n-2) \\
& \times\left\{\frac{(n-3) q_{n}(C)}{40(n-1)}-\frac{(3 n-1)(4 n-3)}{8}\right\}-\frac{272 n^{3}-508 n^{2}+342 n+27}{240} \\
& +\frac{n\left\{\left(20 C^{2}-115 C+88\right) n^{2}-\left(55 C^{2}-377 C+395\right) n+3\left(C^{2}-50 C+50\right)\right\}}{15(3-C)^{2}} \\
& -\frac{n\left(2 n^{3}-4 n^{2}+6 n-3\right)}{12} \\
& =-\frac{40 n^{4}+552 n^{3}-1498 n^{2}+1152 n-153}{240} \\
& -\frac{\left(n^{2}-n+1\right)\left\{3 n(12 n-13) p_{n}+18 n^{2}-28 n-4\right\}}{108}+\frac{(n-2)(n-3) q_{n}(C)}{40(n-1)} \\
& +\frac{n\left\{\left(20 n^{2}-55 n+3\right) C^{2}-\left(115 n^{2}-377 n+150\right) C+88 n^{2}-395 n+150\right\}}{15(3-C)^{2}} \\
& n(n-1)^{n-6}(X-1)^{3}: \frac{\left(n^{2}-n+1\right)(4 n-5) p_{n}\left(6 n p_{n}+18 n^{2}-28 n-4\right)}{216} \\
& +\frac{(n-2)(n-3)(4 n-3) q_{n}(C)}{80(n-1)}-\frac{(n-2)\left(24 n^{3}+224 n^{2}-221 n+39\right)}{160} \\
& -\frac{n(n-2)(n-3)(4 n-3)(4-C)}{6(3-C)^{2}}-\frac{n(2 n-1)\left(4 n^{3}-7 n^{2}+9 n-4\right)}{24} \\
& =-\frac{160 n^{5}-288 n^{4}+1028 n^{3}-2347 n^{2}+1523 n-234}{480} \\
& +\frac{(4 n-5)\left(n^{2}-n+1\right) p_{n}\left(6 n p_{n}+18 n^{2}-28_{n}-4\right)}{216}+(n-2)(n-3)(4 n-3) \\
& \times\left\{\frac{1}{80(n-1)} q_{n}(C)-\frac{(4-C) n}{6(3-C)}\right\} ;
\end{aligned}
$$




$$
n(n-1)^{n-7}(X-1)^{4}:-\frac{3(n-2)(n-3)(4 n-1)\left(8 n^{2}-8 n+3\right)}{320}
$$

Observing these computations, we can obtain easily the inequality (2.12).

Second, we shall investigate the case $4 \leqq n<5$.

Q.E. D.

LEMMA 2.1'. We have

$$
\begin{aligned}
\frac{U_{o}(x)}{\sqrt{n-1}}> & \frac{n^{2}\left(n^{2}-n+1\right)}{6}(n-1)^{n-4}(X-1) \\
& -\frac{n^{2}\left(n^{2}-n+1\right)\left\{(12 n-13) p_{n}+6 n-10\right\}}{36}(n-1)^{n-5}(X-1)^{2} \\
& +\frac{n^{2}(4 n-5)\left(n^{2}-n+1\right) p_{n}\left(p_{n}+3 n-5\right)}{36}(n-1)^{n-6}(X-1)^{3} \\
& \text { for } 1<X \leqq C, 4 \leqq n<5,
\end{aligned}
$$

where $C=1.225, \quad p_{n}=\left(\frac{n-1}{n-1.225}\right)^{n-1.169}$, when $4 \leqq n<4.5 ; \quad C=1.163, \quad p_{n}=$ $\left(\frac{n-1}{n-1.2}\right)^{n-1.165}$, when $4.5 \leqq n<5$, and $X=X_{n}(x)$.

Proof. We obtain this from Lemma 5.1 in (VII).

Q.E. D.

LEMMA $2.3^{\prime}$. We have

$$
\begin{aligned}
& \text { 2.6') } \quad-\frac{U_{1}(x)}{\sqrt{n-1}}>-\frac{n(2 n-1)}{2}(n-1)^{n-3}+\frac{n\left(5 n^{2}-3 n+1\right)}{4}(n-1)^{n-4}(1-x) \\
& +\left\{\frac{n\left(8 n^{3}-50 n^{2}+44 n-9\right)}{16}-\frac{3(n-2)(n-3)(7 n-8)}{40} \frac{(n-1)^{4}}{n^{3}} e_{n-1}\right\}(n-1)^{n-5}(1-x)^{2} \\
& -\frac{n(n-2)(3 n-1)\left(8 n^{2}-n+3\right)}{32}(n-1)^{n-6}(1-x)^{3}+\frac{3(n-2)(n-3)(7 n-8)}{320 n^{3}} e_{n-1}(n-1)^{n-3} \\
& \quad \times\left[4(n-1)(4 n-3)-\left(8 n^{2}-8 n+3\right)(1-x)\right](1-x)^{3} \quad \text { for } 0<x<1,4 \leqq n<5,
\end{aligned}
$$

where $e_{n-1}=\left(1+\frac{1}{n-1}\right)^{n-1}$.

Proof. We obtain this from the proof of Lemma 5.3 in (VII), regarding the last expression in the brackets.

Q.E.D.

LEMMA 2.5'. We have

$$
-\frac{U_{6}(x)}{\sqrt{n-1}}>-n^{2}(n-1)^{n-3}-\frac{n^{2}(8 n-1)}{6}(n-1)^{n-4}(X-1)
$$




$$
\begin{aligned}
& +\left\{\frac{n^{2}(n-2)(13 n-3)}{12}-\frac{n^{2}\left(8 n^{2}-8 C n+3 C^{2}\right)}{8}\left(\frac{n-1}{n-C}\right)^{2.5}\right\}(n-1)^{n-5}(X-1)^{2} \\
& -\frac{n^{2}(n-2)(n-3)(4 n-3)}{8}(n-1)^{n-6}(X-1)^{3} \\
& +n^{2}(n-1)^{n-3} \cdot \frac{8 n^{2}-8 n C+3 C^{2}}{8(n-C)^{2}} \cdot \sqrt{\frac{n-1}{n-C}} \cdot \frac{n-2}{n-1} \cdot\left[\frac{2}{3}-\frac{n-3}{4(n-1)}(X-1)\right](X-1)^{3}, \\
& \text { for } 1<X \leqq C \leqq \min \left(2, b_{n}\right), n \geqq 4 .
\end{aligned}
$$

Proof. We obtain this from the proof of Lemma 5.5 in (VII), regarding the last expression in the brackets.

Proposition 2. We have

$\frac{V\left(x, X_{n}(x)\right)}{(n-1)^{n-3.5}(X-1)}>\frac{n(2 n-1)\left(n^{2}-n-3\right)}{6}$

$$
\begin{aligned}
& -\frac{X-1}{n-1}\left[\frac{16 n^{5}+151 n^{4}-666 n^{3}+1329 n^{2}-1188 n+432}{96}\right. \\
& \quad+\frac{n^{2}\left(n^{2}-n+1\right)\left\{(12 n-13) p_{n}+6 n-10\right\}}{36}-\frac{n(n-2)(n-3)}{40(n-1)} q_{n}(C) \\
& \left.\quad+\frac{n^{2}\left(8 n^{2}-8 n C+3 C^{2}\right)}{8}\left(\frac{n-1}{n-C}\right)^{5 / 2}\right] \\
& -\left(\frac{X-1}{n-1}\right)^{2}\left[\frac{160 n^{6}-264 n^{5}+1898 n^{4}-8485 n^{3}+14555 n^{2}-10386 n+2592}{480}\right. \\
& \quad-\frac{n^{2}(4 n-5)\left(n^{2}-n+1\right) p_{n}\left(p_{n}+3 n-5\right)}{36}-\frac{n(n-2)(n-3)(4 n-3)}{80(n-1)} q_{n}(C) \\
& \left.-\frac{n^{2}(n-2)\left(8 n^{2}-8 n C+3 C^{2}\right)}{12}\left(\frac{n-1}{n-C}\right)^{5 / 2}\right] \\
& -\left(\frac{X-1}{n-1}\right)^{3} \frac{(n-2)(n-3)}{32}\left[\frac{3(n-1)(7 n-8)\left(8 n^{2}-8 n+3\right)}{8}\right. \\
& \left.+n^{2}\left(8 n^{2}-8 n C+3 C^{2}\right)\left(\frac{n-1}{n-C}\right)^{5 / 2}\right] \\
& \text { for } 1<X \leqq C, 4 \leqq n<5,
\end{aligned}
$$

where $X=X_{n}(x), C=1.225, p_{n}=\left(\frac{n-1}{n-1.225}\right)^{n-1.169}$ for $4 \leqq n<4.5 ; C=1.163, p_{n}=$ $\left(\frac{n-1}{\sqrt{n-1.2}}\right)^{n-1.165}$ for $4.5 \leqq n<5$.

Proof. Using Lemma 2.1', Lemma 2.2, Lemma 2.3', Lemma 2.4, Lemma 
$2.5^{\prime}$ and Lemma 2.6 in the expression of $V\left(x, X_{n}(x)\right)$ :

$$
V(x, X(x))=U_{0}(x)+U_{5}(x)-U_{1}(x)+U_{2}(x)-U_{6}(x)+U_{4}(x)
$$

as in the case of $3 \leqq n<4$ and noticing the facts as

the sum of the coefficients of $(n-1)^{n-4}(1-x)$ :

$$
\frac{n\left(5 n^{2}-3 n+1\right)}{4}-\frac{n^{2}(8 n-1)}{6}=-\frac{n\left(n^{2}+7 n-3\right)}{12}<0,
$$

the sum of the coefficients of $(n-1)^{n-5}(1-x)^{2}$ :

$$
\begin{aligned}
& \frac{n\left(8 n^{3}-50 n^{2}+44 n-9\right)}{16}-\frac{3(n-2)(n-3)(7 n-8)}{40} \frac{(n-1)^{4}}{n^{3}} e_{n-1}-\frac{n^{2}(n-2)(4 n-3)}{3} \\
& =-\left\{\frac{n\left(40 n^{3}-26 n^{2}-36 n+27\right)}{48}+\frac{3(n-2)(n-3)(7 n-8)}{40} \frac{(n-1)^{4}}{n^{3}} e_{n-1}\right\}<0,
\end{aligned}
$$

the sum of the coefficients of $(n-1)^{n-6}(1-x)^{3}$ :

$$
\begin{aligned}
& -\frac{n(n-2)(3 n-1)\left(8 n^{2}-8 n+3\right)}{32}+\frac{3(n-2)(n-3)(7 n-8)(4 n-3)(n-1)^{4}}{80 n^{3}} e_{n-1} \\
& =-\frac{n-2}{320}\left\{10 n(3 n-1)\left(8 n^{2}-8 n+3\right)-12 e_{n-1}\left(1-\frac{1}{n}\right)^{3} \cdot(n-1)(n-3)(4 n-3)(7 n-8)\right\}
\end{aligned}
$$

(using $e_{n-1}(1-1 / n)^{3} \leqq e_{4}(1-1 / 5)^{3}=5 / 4$ for $4 \leqq n<5$ )

$$
\begin{aligned}
& \leqq-\frac{n-2}{320}\left\{240 n^{4}-320 n^{3}+170 n^{2}-30 n-15\left(28 n^{4}-165 n^{3}+320 n^{2}-255 n+72\right)\right\} \\
& =-\frac{n-2}{320}\left(-180 n^{4}+2155 n^{3}-4630 n^{2}+3795 n-1080\right)<0
\end{aligned}
$$

(in fact $-180 n^{4}+2155 n^{3}-4630 n^{2}+3795 n-1080 \geqq 1110 n^{2}+3795 n-1080>0$ for $4 \leqq$ $n<5)$, and the coefficient of $(n-1)^{n-7}(1-x)^{4}$ is also negative, we may replace $1-x$ by $X-1$ in these evaluating expressions. Then we compute the coefficients of $(n-1)^{n-3-m}(X-1)^{m}, m=0,1,2,3,4$. The coefficient corresponding to $m=0$ vanishes ;

$$
\begin{aligned}
& (n-1)^{n-4}(X-1): \frac{n(2 n-1)\left(n^{2}-n-3\right)}{6} ; \\
& (n-1)^{n-5}(X-1)^{2}:-\frac{n^{2}\left(n^{2}-n+1\right)\left\{(12 n-13) p_{n}+6 n-10\right\}}{36}+\frac{n(n-2)(n-3) q_{n}(C)}{40(n-1)} \\
& \quad-\frac{n(n-2)(3 n-1)(4 n-3)}{8}-\frac{n\left(40 n^{3}-26 n^{2}-36 n+27\right)}{48} \\
& \quad-\frac{3(n-2)(n-3)(7 n-8)(n-1)^{4}}{40 n^{3}} e_{n-1}+\frac{n^{2}(n-2)(13 n-3)}{12}
\end{aligned}
$$




$$
\begin{aligned}
& -\frac{n^{2}\left(8 n^{2}-8 n C+3 C^{2}\right)}{8}\left(\frac{n-1}{n-C}\right)^{5 / 2}-\frac{n^{2}\left(2 n^{3}-4 n^{2}+6 n-3\right)}{12} \\
= & -\frac{n\left(8 n^{4}+44 n^{3}-108 n^{2}+102 n-9\right)}{48}-\frac{n\left(n^{2}-n+1\right)\left\{(12 n-13) p_{n}+6 n-10\right\}}{36} \\
& +\frac{n(n-2)(n-3) q_{n}(C)}{40(n-1)}-\frac{n^{2}\left(8 n^{2}-8 n C+3 C^{2}\right)}{8}\left(\frac{n-1}{n-C}\right)^{5 / 2} \\
& -\frac{3(n-2)(n-3)(7 n-8)}{40} \frac{(n-1)^{4} e_{n-1}}{n^{3}},
\end{aligned}
$$

regarding the last term, using the inequality $\frac{(n-1)^{3} e_{n-1}}{n^{3}}<\frac{5}{4}$ for $4 \leqq n<5$, we have

$$
\begin{aligned}
& \frac{n\left(8 n^{4}+44 n^{3}-108 n^{2}+102 n-9\right)}{48}+\frac{3(n-2)(n-3)(7 n-8)(n-1)^{4} e_{n-1}}{40 n^{3}} \\
& <\frac{n\left(8 n^{4}+44 n^{3}-108 n^{2}+102 n-9\right)}{48}+\frac{3}{32} \cdot(n-1)(n-2)(n-3)(7 n-8) \\
& =\frac{16 n^{5}+88 n^{4}-216 n^{3}+204 n^{2}-18 n+9\left(7 n^{4}-50 n^{3}+125 n^{2}-130 n+48\right)}{96} \\
& =\frac{16 n^{5}+151 n^{4}-666 n^{3}+1329 n^{2}-1188 n+432}{96},
\end{aligned}
$$

hence we can replace the last expression by the following smaller one as follows :

$$
\begin{aligned}
& -\frac{16 n^{5}+151 n^{4}-666 n^{3}+1329 n^{2}-1188 n+432}{96}-\frac{n^{2}\left(n^{2}-n+1\right)\left\{(12 n-13) p_{n}+6 n-10\right\}}{36} \\
& +\frac{n(n-2)(n-3) q_{n}(C)}{40(n-1)}-\frac{n^{2}\left(8 n^{2}-8 n C+3 C^{2}\right)}{8}\left(\frac{n-1}{n-C}\right)^{5 / 2} ; \\
& (n-1)^{n-6}(X-1)^{3}: \frac{n^{2}(4 n-5)\left(n^{2}-n+1\right) p_{n}\left(p_{n}+3 n-5\right)}{36}+\frac{n(n-2)(n-3)(4 n-3) q_{n}(C)}{80(n-1)} \\
& -\frac{n(n-2)(3 n-1)\left(8 n^{2}-8 n+3\right)}{32}+\frac{3(n-1)(n-2)(n-3)(4 n-3)(7 n-8)}{80} \frac{(n-1)^{3} e_{n-1}}{n^{3}} \\
& -\frac{n^{2}(n-2)(n-3)(4 n-3)}{8}+\frac{2}{3} \frac{n^{2}(n-1)^{3}\left(8 n^{2}-8 n C+3 C^{2}\right)}{8(n-C)^{2}} \cdot \sqrt{\frac{n-1}{n-C} \cdot \frac{n-2}{n-1}} \\
& -\frac{n^{2}(2 n-1)\left(4 n^{3}-7 n^{2}+9 n-4\right)}{24} \\
& =-\frac{n\left(32 n^{5}+48 n^{4}-416 n^{3}+643 n^{2}-311 n+18\right)}{96}+\frac{3(n-1)(n-2)(n-3)(4 n-3)(7 n-8)}{80} \\
& \times \frac{(n-1)^{3} e_{n-1}}{n^{3}}
\end{aligned}
$$




$$
\begin{aligned}
& +\frac{n^{2}(4 n-5)\left(n^{2}-n+1\right) p_{n}\left(p_{n}+3 n-5\right)}{36}+\frac{n(n-2)(n-3)(4 n-3) q_{n}(C)}{80(n-1)} \\
& +\frac{n^{2}(n-2)\left(8 n^{2}-8 n C+3 C^{2}\right)}{12}\left(\frac{n-1}{n-C}\right)^{5 / 2}
\end{aligned}
$$

(using $(n-1)^{3} e_{n-1} / n^{3}>(3 / 4)^{3} e_{3}=1$ for $4 \leqq n<5$, the sum of the first two terms can be replaced by

$$
\begin{aligned}
& >-\frac{n\left(32 n^{5}+48 n^{4}-416 n^{3}+643 n^{2}-311 n+18\right)}{96}+\frac{3(n-1)(n-2)(n-3)(4 n-3)(7 n-8)}{80} \\
& =-\frac{5\left(32 n^{6}+48 n^{5}-416 n^{4}+643 n^{3}-311 n^{2}+18 n\right)-18\left(28 n^{5}-221 n^{4}+650 n^{3}-895 n^{2}+582 n-144\right)}{480} \\
& \left.=-\frac{160 n^{6}-264 n^{5}+1898 n^{4}-8485 n^{3}+14555 n^{2}-10386 n+2592}{480}\right) ; \\
& (n-1)^{n-7}(X-1)^{4}:-\frac{3(n-2)(n-3)(7 n-8)\left(8 n^{2}-8 n+3\right)}{320} \frac{(n-1)^{4} e_{n-1}}{n^{3}} \\
& \quad-\frac{n^{2}(n-2)(n-3)\left(8 n^{2}-8 n C+3 C^{2}\right)}{32}\left(\frac{n-1}{n-C}\right)^{5 / 2}
\end{aligned}
$$

(using $(n-1)^{3} e_{n-1} / n^{3}<5 / 4$ for $4 \leqq n<5$ )

$$
\begin{aligned}
> & -\frac{3}{256}(n-1)(n-2)(n-3)(7 n-8)\left(8 n^{2}-8 n+3\right) \\
& -\frac{1}{32} n^{2}(n-2)(n-3)(8 n-8 n C+3 C)\left(\frac{n-1}{n-C}\right)^{5 / 2} .
\end{aligned}
$$

Using the above computed results, we obtain the inequality in the statement.

Q.E.D.

§3. Concrete evaluation of $V\left(x, X_{n}(x)\right)$ near $x=1$ in case $4 \leqq n<5$.

In this section, we shall discuss more concretely the content of Proposition 2. We consider the case, by dividing into the two cases $4.5 \leqq n<5$ and $4 \leqq n<4.5$.

First of all, we shall prove the following lemma in order to evaluate $p_{n}$.

LEMMA 3.1. Let $1<a<b<2 a-1$, then $\left(\frac{n-1}{n-b}\right)^{n-a}$ is increasing with respect to $n$ in $\max \left\{b, \frac{a b+a-2 b}{2 a-b-1}\right\}<n<+\infty$.

Proof. Differentiating logarithmically the function of $n$, we obtain

$$
\log \frac{n-1}{n-b}-\frac{(b-1)(n-a)}{(n-1)(n-b)} \quad(\rightarrow 0 \text { as } n \rightarrow+\infty),
$$

whose derivative is 


$$
\begin{aligned}
& -\frac{b-1}{(n-1)(n-b)}-\frac{(b-1)\{(n-1)(n-b)-(n-a)(2 n-b-1)\}}{(n-1)^{2}(n-b)^{2}} \\
& =-\frac{(b-1)\{(2 a-b-1) n-(a b+a-2 b)\}}{(n-1)^{2}(n-b)^{2}},
\end{aligned}
$$

which is negative for $n>(a b+a-2 b) /(2 a-b-1)$. From these we obtain the statement. We see easily that $a b+a-2 b>0$.

Q.E. D.

Now, we shall consider the case $4.5 \leqq n<5$. Let $C=1.163$ and $p_{n}=$ $\left(\frac{n-1}{n-1.2}\right)^{n-1.165}$. Then, we see that, setting $a=1.165, b=1.2$ in Lemma 3.1, they satisfy the condition: $1<a<b<2 a-1$, and we have

$$
2 a-b-1=0.13 \text { and } a b+a-2 b=0.163
$$

and so $p_{n}$ is increasing in $0.163 / 0.13 \doteq 1.2538<n<+\infty$. Hence, we obtain for $4.5 \leqq n<5$

$$
\begin{aligned}
& p_{n}<(4 / 3.8)^{3.835}=1.2173906 \cdots<1.2174, \\
& p_{n} \geqq(3.5 / 3.3)^{3.335}=1.2168104 \cdots>1.2168, \\
& p_{n}^{2} \geqq(3.5 / 3.3)^{6.67}=1.4806277 \cdots>1.4806 .
\end{aligned}
$$

We obtain from (2.5)

$$
\begin{aligned}
q_{n}(C) & =n^{2}\left(C^{2}-10 C+21\right)+n(9 C-24)-C^{2}+4 C \\
& =n^{2} \times 10.722569-n \times 13.533+3.299431 \\
& >10.7225 n^{2}-13.533 n+3.2994 .
\end{aligned}
$$

We have also

and

$$
\left(\frac{n-1}{n-C}\right)^{5 / 2}=\left(\frac{n-1}{n-1.163}\right)^{5 / 2}\left\{\begin{array}{l}
\leqq(3.5 / 3.337)^{5 / 2}=1.1266255 \cdots<1.1267 \\
>(4 / 3.837)^{5 / 2}=1.1096103 \cdots>1.1096
\end{array}\right.
$$

$$
8 n^{2}-8 n C+3 C^{2}=8 n^{2}-9.304 n+4.057707\left\{\begin{array}{l}
>8 n^{2}-9.304 n+4.0577 \\
<8 n^{2}-9.304 n+4.0578 .
\end{array}\right.
$$

Using these evaluations in the right hand side of the inequality (2.13) and taking account of the signs of the expressions in it, we obtain

$$
\begin{gathered}
\frac{V\left(x, X_{n}(x)\right)}{(n-1)^{n-3.5}(X-1)}>\frac{n(2 n-1)\left(n^{2}-n-3\right)}{6} \\
-\frac{X-1}{n-1}\left[\frac{16 n^{5}+151 n^{4}-666 n^{3}+1329 n^{2}-1188 n+432}{96}\right. \\
\quad+\frac{n^{2}\left(n^{2}-n+1\right)(20.6088 n-25.8262)}{36}
\end{gathered}
$$


290

A CERTAIN PROPERTY OF GEODESICS OF $O_{n}^{2}$

$$
\begin{gathered}
-\frac{n(n-2)(n-3)\left(10.7225 n^{2}-13.533 n+3.2994\right)}{40(n-1)} \\
\left.+\frac{n^{2}\left(8 n^{2}-9.304 n+4.0578\right) \times 1.1267}{8}\right] \\
-\left(\frac{X-1}{n-1}\right)^{2}\left[\frac{160 n^{6}-264 n^{5}+1898 n^{4}-8485 n^{3}+14555 n^{2}-10386 n+2592}{480}\right. \\
-\frac{n^{2}(4 n-5)\left(n^{2}-n+1\right)(3.6504 n-4.6034)}{36} \\
-\frac{n(n-2)(n-3)(4 n-3)\left(10.7225 n^{2}-13.533 n+3.2994\right)}{80(n-1)} \\
\left.-\frac{n^{2}(n-2)\left(8 n^{2}-9.304 n+4.0577\right) \times 1.1096}{12}\right] \\
-\left(\frac{X-1}{n-1}\right)^{3} \frac{(n-2)(n-3)\left(240.1088 n^{4}-611.8625 n^{3}+651.5754 n^{2}-327 n+72\right)}{256} \quad \text { for } 1<X \leqq 1.163 .
\end{gathered}
$$

We can see the properties of the expressions in (2.13), which are necessary in the process of replacements regarding the above evaluations, in the proof of Theorem 2 in [20]. Here, we show only the evaluation of the coefficient of $\left(\frac{X-1}{n-1}\right)^{3}$. In fact, we have

$$
\begin{aligned}
& 3(n-1)(7 n-8)\left(8 n^{2}-8 n+3\right)+8 n^{2}\left(8 n^{2}-8 n C+3 C^{2}\right)\left(\frac{n-1}{n-C}\right)^{5 / 2} \\
& <3(n-1)(7 n-8)\left(8 n^{2}-8 n+3\right)+8 n^{2}\left(8 n^{2}-9.304 n+4.0578\right) \times 1.1267 \\
& =3\left(56 n^{4}-176 n^{3}+205 n^{2}-109 n+24\right)+9.0136\left(8 n^{4}-9.304 n^{3}+4.0578 n^{2}\right) \\
& =240.1088 n^{4}-611.8625344 n^{3}+651.5753861 n^{2}-327 n+72 \\
& <240.1088 n^{4}-611.8625 n^{3}+651.5754 n^{2}-327 n+72 .
\end{aligned}
$$

In order to proceed the argument, we evaluate the value of $t=\frac{X-1}{n-1}$, which makes the right hand side of (3.1) as a cubic polynomial of $t$ positive at $n=5$ and $n=4.5$.

Observation $1(n=5)$. We have

$$
\frac{n(2 n-1)\left(n^{2}-n-3\right)}{6}=\frac{5 \times 9 \times 17}{6}=127.5 \text {, }
$$

the coefficient of $-t=\frac{88842}{96}+\frac{25 \times 21 \times 77.2178}{36}-\frac{5 \times 3 \times 2 \times 203.6969}{10 \times 4}$ 


$$
\begin{aligned}
& +\frac{\mid 25 \times 157.5378 \times 1.1267}{8} \doteqdot 925.4375+1126.092917-38.19316875+554.6807477 \\
& \doteqdot 2568.017996 \text {, } \\
& \text { the - coefficient of }-t^{2}=\frac{2115162}{480}-\frac{25 \times 15 \times 21 \times 13.6486}{36}-\frac{5 \times 3 \times 2 \times 17 \times 203.6969}{80 \times 4} \\
& -\frac{25 \times 3 \times 157.5377 \times 1.1096}{12} \doteq 4406.5875-2985.63125-324.6419344-1092.52395 \\
& \doteqdot 3.7903656 \text {, }
\end{aligned}
$$

the coefficient of $-t^{3}=\frac{3 \times 2 \times 88311.5725}{256}=2069.80248$,

and so

the right hand side of (3.1) with $n=5$

$>127.5-2568.018 t-3.7904 t^{2}-2069.8025 t^{3}$,

which becomes $0.12278 \cdots$ at $t=0.0495$ and $-0.13558 \cdots$ at $t=0.0496$.

Observation $2(n=4.5)$. We have

$$
\frac{n(2 n-1)\left(n^{2}-n-3\right)}{6}=\frac{4.5 \times 8 \times 12.75}{6}=76.5 \text {, }
$$

the coefficient of $-t=\frac{52752.9375}{96}+\frac{4.5 \times 4.5 \times 16.75 \times 66.9134}{36}$

$$
\begin{aligned}
& -\frac{4.5 \times 2.5 \times 1.5 \times 159.531525}{40 \times 3.5}+\frac{4.5 \times 4.5 \times 124.1898 \times 1.1267}{8}=549.5097656 \\
& +630.4496906-19.22924632+354.1842644 \doteqdot 1514.914474,
\end{aligned}
$$

the coefficient of $-t^{2}=\frac{1097145}{480}-\frac{4.5 \times 4.5 \times 13 \times 16.75 \times 11.8234}{36}$

$$
\begin{aligned}
& -\frac{4.5 \times 2.5 \times 1.5 \times 15 \times 159.531525}{80 \times 3.5}-\frac{4.5 \times 4.5 \times 2.5 \times 124.1897 \times 1.1096}{12} \doteqdot 2285.71875 \\
& -1448.181759-144.2193474-581.3475094 \doteqdot 111.9701342,
\end{aligned}
$$

the coefficient of $-t^{3}=\frac{2.5 \times 1.5 \times 54498.54634}{256} \doteq 798.3185499$,

and so

the right hand side of (3.1) with $n=4.5$

$$
>76.5-1514.9145 t-111.9702 t^{2}-798.3186 t^{3} \text {, }
$$

which becomes $0.06813 \cdots$ at $t=0.0502$ and $-0.08509 \cdots$ at $t=0.0503$.

Connecting linearly the two points $(4.5,0.0502)$ and $(5,0.0495)$ on the 
$(n, t)$-plane by

$$
t=(565-14 n) / 10000 \text {, }
$$

we have the following proposition which is proved in another paper [20].

Proposition 3. When $4.5 \leqq n<5$, the cubic polynomial of $t$ :

$$
\begin{aligned}
& \frac{n(2 n-1)\left(n^{2}-n-3\right)}{6} \\
& -t\left[\frac{16 n^{5}+151 n^{4}-666 n^{3}+1329 n^{2}-1188 n+432}{96}\right. \\
& \quad+\frac{n^{2}\left(n^{2}-n+1\right)(20.6088 n-25.8262)}{36} \\
& \quad-\frac{n(n-2)(n-3)\left(10.7225 n^{2}-13.533 n+3.2994\right)}{40(n-1)} \\
& \left.\quad+\frac{n^{2}\left(8 n^{2}-9.304 n+4.0578\right) \times 1.1267}{8}\right] \\
& -t^{2}\left[\frac{160 n^{6}-264 n^{5}+1898 n^{4}-8485 n^{3}+14555 n^{2}-10386 n+2592}{480}\right. \\
& \quad-\frac{n^{2}(4 n-5)\left(n^{2}-n+1\right)(3.6504 n-4.6034)}{36} \\
& \quad-\frac{n(n-2)(n-3)(4 n-3)\left(10.7225 n^{2}-13.533 n+3.2994\right)}{80(n-1)} \\
& \left.\quad-\frac{n^{2}(n-2)\left(8 n^{2}-9.304 n+4.0577\right) \times 1.1096}{12}\right] \\
& -t^{3} \frac{(n-2)(n-3)\left(240.1088 n^{4}-611.8625 n^{3}+651.5754 n^{2}-327 n+72\right)}{256}
\end{aligned}
$$

is positive for $0 \leqq t \leqq(565-14 n) / 10000$.

Next, we shall consider the case $4 \leqq n<4.5$. Let $C=1.225$ and $p_{n}=$ $\left(\frac{n-1}{n-1.225}\right)^{n-1.169}$. Then, we see that setting $a=1.169, b=1.225$ in Lemma 3.1, they satisfy the condition $1<a<b<2 a-1$, and we have

$$
2 a-b-1=0.113 \text { and } a b+a-2 b=0.151025
$$

and so $p_{n}$ is increasing in $0.151025 / 0.113 \doteqdot 1.3365<n<+\infty$. Hence we obtain for $4 \leqq n<4.5$ 


$$
\begin{aligned}
& p_{n}<\left(\frac{3.5}{3.275}\right)^{3.331}=1.2477334 \cdots<1.2478, \\
& p_{n} \geqq\left(\frac{3}{2.775}\right)^{2.831}=1.2469606 \cdots>1.2469, \\
& p_{n}^{2} \geqq\left(\frac{3}{2.775}\right)^{5.662}=1.5549109 \cdots>1.5549 .
\end{aligned}
$$

We obtain from (2.5)

$$
\begin{aligned}
q_{n}(C) & =n^{2}\left(C^{2}-10 C+21\right)+n(9 C-24)-C^{2}+4 C \\
& =n^{2} \times 10.250625-n \times 12.975+3.399375 \\
& >10.2506 n^{2}-12.975 n+3.3993 .
\end{aligned}
$$

We have also

$$
\left(\frac{n-1}{n-C}\right)^{5 / 2}=\left(\frac{n-1}{n-1.225}\right)^{5 / 2}\left\{\begin{array}{l}
\leqq(3 / 2.775)^{5 / 2}=1.2151941 \cdots<1.2152 \\
>(3.5 / 3.275)^{5 / 2}=1.1807062 \cdots>1.1807
\end{array}\right.
$$

and

$$
8 n^{2}-8 n C+3 C^{2}=8 n^{2}-9.8 n+4.501875\left\{\begin{array}{l}
>8 n^{2}-9.8 n+4.5018 \\
<8 n^{2}-9.8 n+4.5019 .
\end{array}\right.
$$

Using these evaluations in the right hand side of the inequality (2.13) and taking account of the signs of the expressions in it, we obtain

$$
\begin{gathered}
\frac{V\left(x, X_{n}(x)\right)}{(n-1)^{n-3.5}(X-1)}>\frac{n(2 n-1)\left(n^{2}-n-3\right)}{6} \\
-\frac{X-1}{n-1}\left[\frac{16 n^{5}+151 n^{4}-666 n^{3}+1329 n^{2}-1188 n+432}{96}\right. \\
+\frac{n^{2}\left(n^{2}-n+1\right)(20.9736 n-26.2214)}{36} \\
\quad-\frac{n(n-2)(n-3)\left(10.2506 n^{2}-12.975 n+3.3993\right)}{40(n-1)} \\
\left.\quad+\frac{n^{2}\left(8 n^{2}-9.8 n+4.5019\right) \times 1.2152}{8}\right] \\
-\left(\frac{X-1}{n-1}\right)^{2}\left[\frac{160 n^{6}-264 n^{5}+1898 n^{4}-8485 n^{3}+14555 n^{2}-10386 n+2592}{480}\right. \\
-\frac{n^{2}(4 n-5)\left(n^{2}-n+1\right)(3.7408 n-4.6799)}{36} \\
-\frac{n(n-2)(n-3)(4 n-3)\left(10.2506 n^{2}-12.975 n+3.3993\right)}{80(n-1)}
\end{gathered}
$$




$$
\begin{gathered}
\left.-\frac{n^{2}(n-2)\left(8 n^{2}-9.8 n+4.5018\right) \times 1.1807}{12}\right] \\
-\left(\frac{X-1}{n-1}\right)^{3} \frac{(n-2)(n-3)\left(245.7728 n^{4}-628.2716 n^{3}+658.7657 n^{2}-327 n+72\right)}{256} \quad \text { for } 1<X \leqq 1.225 .
\end{gathered}
$$

In the above procession, we have also used the facts proved in the proof of Theorem 3 in [20]. Here, we show only the evaluation of $p_{n}\left(p_{n}+3 n-5\right)$ as follows:

$$
\begin{aligned}
p_{n}\left(p_{n}+3 n-5\right) & =p_{n}^{2}+p_{n}(3 n-5) \\
& >1.5549+1.24696(3 n-5)>3.7408 n-4.6799 .
\end{aligned}
$$

In order to proceed with the argument, we evaluate the value of $t=(X-1)$ $/(n-1)$, which makes the right hand side of (3.3) as a cubic polynomial of $t$ positive at $n=4.5$ and $n=4$.

Observation $3(n=4.5)$. Using partially the computations in Observation 2, we have

$$
\frac{n(2 n-1)\left(n^{2}-n-3\right)}{6}=76.5 \text {, }
$$

the coefficient of $-t=\frac{52752.9375}{96}+\frac{4.5 \times 4.5 \times 16.75 \times 68.1598}{36}$

$$
\begin{aligned}
& -\frac{4.5 \times 2.5 \times 1.5 \times 152.58645}{40 \times 3.5}+\frac{4.5 \times 4.5 \times 122.4019 \times 1.2152}{8} \doteq 549.5097656 \\
& +642.1931156-18.39211674+376.5051844 \doteqdot 1549.815949,
\end{aligned}
$$

the coefficient of $-t^{2}=\frac{1097145}{480}-\frac{4.5 \times 4.5 \times 13 \times 16.75 \times 12.1537}{36}$

$$
\begin{aligned}
& -\frac{4.5 \times 2.5 \times 1.5 \times 15 \times 152.58645}{80 \times 3.5}-\frac{4.5 \times 4.5 \times 2.5 \times 122.4018 \times 1.1807}{12} \doteqdot 2285.71875 \\
& -1488.638348-137.9408756-609.6929284 \doteqdot 49.446598,
\end{aligned}
$$

the coefficient of $-t^{3}=\frac{2.5 \times 1.5 \times 55471.46468}{256} \doteqdot 812.5702834$,

and so

the right hand side of (3.3) with $n=4.5$

$$
>76.5-1549.816 t-49.4466 t^{2}-812.5703 t^{3},
$$

which becomes $0.032586 \cdots$ at $t=0.0492$ and $-0.123473 \cdots$ at $t=0.0493$.

Observation $4(n=4)$. We have 


$$
\frac{n(2 n-1)\left(n^{2}-n-3\right)}{6}=\frac{4 \times 7 \times 9}{6}=42 \text {, }
$$

the coefficient of $-t=\frac{29360}{96}+\frac{16 \times 13 \times 57.673}{36}-\frac{4 \times 2 \times 1 \times 115.5089}{40 \times 3}$

$$
\begin{aligned}
+\frac{16 \times 93.3019 \times 1.2152}{8} & \doteq 305.8333333+333.2217778-7.0059333+226.7609378 \\
& \doteq 858.8101156,
\end{aligned}
$$

the coefficient of $-t^{2}=\frac{521800}{480}-\frac{16 \times 11 \times 13 \times 10.2833}{36}-\frac{4 \times 2 \times 13 \times 115.5089}{80 \times 3}$

$$
\begin{aligned}
-\frac{16 \times 2 \times 93.3018 \times 1.1807}{12} & =1087.0833333-653.5608444-50.0538566-293.7638274 \\
& \doteq 89.7048046,
\end{aligned}
$$

the coefficient of $-t^{3}=\frac{2 \times 1 \times 32012.7056}{256}=250.0992625$

and so

the right hand side of (3.3) with $n=4$

$$
>42-858.8102 t-89.7049 t^{2}-250.0993 t^{3} \text {, }
$$

which becomes $0.021235 \cdots$ at $t=0.0486$ and $-0.065695 \cdots$ at $t=0.0487$.

Connecting linearly the two points $(4,0.0486)$ and $(4.5,0.0492)$ on the $(n, t)$-plane by

$$
t=(12 n+438) / 10000=6(2 n+73) / 10000,
$$

we have the following proposition which is proved also in [20].

PROPOSITION 4. When $4 \leqq n<4.5$, the cubic polynomial of $t$ :

$$
\begin{aligned}
& \frac{n(2 n-1)\left(n^{2}-n-3\right)}{6} \\
& -t\left[\frac{16 n^{5}+151 n^{4}-666 n^{3}+1329 n^{2}-1188 n+432}{96}\right. \\
& \quad+\frac{n^{2}\left(n^{2}-n+1\right)(20.9736 n-26.2214)}{36} \\
& \quad-\frac{n(n-2)(n-3)\left(10.2506 n^{2}-12.975 n+3.3993\right)}{40(n-1)} \\
& \left.\quad+\frac{n^{2}\left(8 n^{2}-9.8 n+4.5019\right) \times 1.2152}{8}\right]
\end{aligned}
$$




$$
\begin{aligned}
-t^{2} & {\left[\frac{160 n^{6}-264 n^{5}+1898 n^{4}-8485 n^{3}+14555 n^{2}-10386 n+2592}{480}\right.} \\
& -\frac{n^{2}(4 n-5)\left(n^{2}-n+1\right)(3.7408 n-4.6799)}{36} \\
& -\frac{n(n-2)(n-3)(4 n-3)\left(10.2506 n^{2}-12.975 n+3.3993\right)}{80(n-1)} \\
& \left.-\frac{n^{2}(n-2)\left(8 n^{2}-9.8 n+4.5018\right) \times 1.1807}{12}\right] \\
-t^{3} \frac{(n-2)(n-3)\left(245.7728 n^{4}\right.}{\left.628.2716 n^{3}+658.7657 n^{2}-327 n+72\right)} & 256
\end{aligned}
$$

is positive for $0 \leqq t \leqq 6(2 n+73) / 10000$.

$\S 4$. Concrete evaluation of $V\left(x, X_{n}(x)\right)$ near $x=1$ in case $3 \leqq n<4$.

In this section, we shall discuss more concretely the content of Proposition 1. For simplicity we set

$$
\begin{aligned}
& A:=\frac{(2 n-1)\left(n^{2}-n-3\right)}{6}, \quad K:=\frac{40 n^{4}+552 n^{3}-1498 n^{2}+1152 n-153}{240}, \\
& F:=\frac{\left(n^{2}-n+1\right)\left\{3 n(12 n-13) p_{n}+18 n^{2}-28 n-4\right\}}{108}, \quad G:=\frac{(n-2)(n-3) q_{n}(C)}{40(n-1)} \\
& H:=\frac{n\left\{\left(20 n^{2}-55 n+13\right) C^{2}-\left(115 n^{2}-377 n+150\right) C+88 n^{2}-395 n+150\right\}}{15(3-C)^{2}} \\
& L:=\frac{160 n^{5}-288 n^{4}+1028 n^{3}-2347 n^{2}+1523 n-234}{480}, \\
& B:=\frac{(4 n-5)\left(n^{2}-n+1\right) p_{n}\left(6 n p_{n}+18 n^{2}-28 n-4\right)}{216}, \\
& J:=\frac{(n-2)(n-3)(4 n-3) q_{n}(C)}{80(n-1)}, \quad D:=\frac{(4-C) n(n-2)(n-3)(4 n-3)}{6(3-C)^{2}} \\
& E:=\frac{3(n-2)(n-3)(4 n-1)\left(8 n^{2}-8 n+3\right)}{320},
\end{aligned}
$$

which are only effective in this section.

Taking account of Observation 4, we assume $C=1.1$ for $n=3$. Then, we have by (1.17), (2.2) and (2.5)

$$
k_{3}(C)=\frac{2+C-\sqrt{C^{2}-4 C+7}}{C}, \quad k_{2}(1.1)=\frac{3.1-\sqrt{3.81}}{1.1}=1.0437071 \cdots,
$$




$$
\begin{aligned}
p_{3} & =2^{3-k} /(3-k)(3-1.1)^{2-k}=(2 /(3-1.0437)) \times(20 / 19)^{2-1.0437} \\
& =(2 / 1.9563) \cdot(20 / 19)^{0.9563}=1.0737358 \cdots,
\end{aligned}
$$

and

$$
q_{3}(C)=8 C^{2}-59 C+117, \quad q_{3}(1.1)=61.78 .
$$

Furthermore, using the above evaluation of $p_{3}$, we obtain

$$
\begin{aligned}
& 3 n(12 n-13) p_{n}+18 n^{2}-28 n-4=207 p_{3}+74 \doteqdot 296.2633226, \\
& p_{n}\left(6 n p_{n}+18 n^{2}-28 n-4\right)=p_{3}\left(18 p_{3}+74\right)=100.208803 .
\end{aligned}
$$

Using these evaluations, we obtain

$$
\begin{aligned}
& A=2.5, \quad K=7965 / 240=33.1875, \quad F=7 \times 296.2633226=19.2022523, \\
& G=0, \quad H=\frac{3\left(18 C^{2}-54 C-243\right)}{15(3-C)^{2}}=-\frac{3 \times 280.62}{15 \times 1.9 \times 1.9} \doteqdot-15.5468144, \\
& L=26520 / 480=55.25, \quad B=7 \times 7 \times 100.208803 / 216 \doteq 22.7325525, \\
& J=0, \quad D=0, \quad E=0,
\end{aligned}
$$

and

$$
\begin{aligned}
& K+F-G-H=33.1875+19.20225+15.54681=67.93656, \\
& L-B-J+D \doteqdot 55.25-22.73255=32.51745 .
\end{aligned}
$$

Using these calculated values, we consider the quadratic polynomial of $t$ :

$$
2.5-67.9366 t-32.5175 t^{2}
$$

which becomes $0.0051116 \cdots$ at $t=0.0361$ and $-0.0019171 \cdots$ at $t=0.0362$.

Taking account of these facts for $n=3$ and $n=4$, we may assume

$$
C=1+\frac{n-1}{20}=\frac{n+19}{20} \quad \text { for } \quad 3 \leqq n<4 \text {. }
$$

Then, we obtain from (2.5) and (1.17)

$$
\begin{gathered}
q_{n}(C)=\frac{1}{400}\left(n^{4}-162 n^{3}+5140 n^{2}-6138 n+1159\right), \\
(n-1) C^{2}-2(n+1) C+5 n-1=\frac{1}{400}\left(n^{3}-3 n^{2}+1523 n-1521\right) \\
=\frac{(n-1)\left(n^{2}-2 n+1521\right)}{400}, \\
k_{n}(C)=\frac{n^{2}+38 n+1-(n-1) \sqrt{n^{2}-2 n+1521}}{2(n+19)}:=k_{n}
\end{gathered}
$$




$$
n-k_{n}(C)=\frac{(n-1)\left(n+1+\sqrt{\left.n^{2}-2 n+1521\right)}\right.}{2(n+19)}:=s_{n} \text {. }
$$

Next from (2.2) we obtain

i. e.

$$
p_{n}=\frac{(n-1)^{n-k}}{(n-k)(n-C)^{n-k-1}}=\frac{n-1}{n-k}\left(\frac{n-1}{n-\frac{n+19}{20}}\right)^{n-k-1},
$$

$$
p_{n}=\frac{n-1}{s_{n}}\left(\frac{20}{19}\right)^{s_{n-1}}
$$

Since we have

$$
\begin{aligned}
& \left(20 n^{2}-55 n+3\right) C^{2}-\left(115 n^{2}-377 n+150\right) C+88 n^{2}-395 n+150 \\
& =\left(20 n^{2}-55 n+3\right)\left(\frac{(n-1)^{2}}{400}+\frac{n-1}{10}+1\right)-\left(115 n^{2}-377 n+150\right)\left(\frac{n-1}{20}+1\right) \\
& \quad+88 n^{2}-395 n+150=\frac{20 n^{4}-1595 n^{3}+4173 n^{2}-37481 n+4083}{400}
\end{aligned}
$$

and $3-C=(41-n) / 20$, we obtain

$$
\begin{aligned}
H & =\frac{n\left\{\left(20 n^{2}-55 n+3\right) C^{2}-\left(115 n^{2}-377 n+150\right) C+88 n^{2}-395 n+150\right\}}{15(3-C)^{2}} \\
& =\frac{n\left(20 n^{4}-1595 n^{3}+4173 n^{2}-37481 n+4083\right)}{15(41-n)^{2}} .
\end{aligned}
$$

We have also

$$
\frac{4-C}{6(3-C)^{2}}=\frac{400}{6(41-n)^{2}}\left(3-\frac{n-1}{20}\right)=\frac{10(61-n)}{3(41-n)^{2}} .
$$

Using these facts, $G, M$ and $D$ can be written as

$$
\begin{aligned}
& G=\frac{(n-2)(n-3)\left(n^{4}-162 n^{3}+5140 n^{2}-6138 n+1159\right)}{16000(n-1)}, \\
& J=\frac{(n-2)(n-3)(4 n-3)\left(n^{4}-162 n^{3}+5140 n^{2}-6138 n+1159\right)}{32000(n-1)}, \\
& D=\frac{10 n(n-2)(n-3)(4 n-3)(61-n)}{3(41-n)^{2}} .
\end{aligned}
$$

Observation $5(n=4)$. We shall compute the values of $K \sim E$ in (4.1) for $n=4$. We have

$$
s_{4}=\frac{3(5+\sqrt{1529})}{2 \times 23}=2.87624540 \cdots,
$$




$$
\begin{aligned}
& p_{4}=\frac{3}{2.8762454}\left(\frac{20}{19}\right)^{1.8762454} \doteqdot 1.1483950, \\
& A=7 \times 9 / 6=10.5, \quad K=26055 / 240=108.5625, \\
& F=\frac{13 \times\left(12 \times 35 \times p_{4}+172\right)}{108}=\frac{13 \times\left(105 p_{4}+43\right)}{27} \doteqdot 78.7614509, \\
& G=\frac{2 \times 1 \times 48735}{3 \times 16000}=2.030625, \quad H=\frac{4 \times(-176033)}{15 \times 37 \times 37} \doteq-34.2893596, \\
& L=124210 / 480=258.7708333, \\
& B=\frac{11 \times 13 \times p_{4} \times\left(24 p_{4}+172\right)}{216}=\frac{143 p_{4}\left(6 p_{4}+43\right)}{54} \doteqdot 151.7226093, \\
& J=\frac{2 \times 1 \times 13 \times 48735}{3 \times 32000}=13.1990625, \quad D=\frac{10 \times 4 \times 2 \times 13 \times 57}{3 \times 37 \times 37} \doteqdot 14.4338933, \\
& E=\frac{3 \times 2 \times 1 \times 15 \times 99}{320}=27.84375
\end{aligned}
$$

and

$$
\begin{aligned}
& K+F-G-H \doteqdot 108.5625+78.7614509-2.030625+34.2893596=219.5826855 \\
& L-B-J+D \doteqdot 258.7708333-151.7226093-13.1990625+14.4338933=108.2830548 .
\end{aligned}
$$

Using these calculated values, we consider the cubic polynomial of $t$ corresponding to the right hand side of (2.12) as follows

$$
10.5-219.58269 t-108.28306 t^{2}-27.84375 t^{3},
$$

which becomes $0.0064991 \cdots$ at $t=0.0467$ and $-0.0164898 \cdots$ at $t=0.0468$.

Now, connecting linearly the two points $(3,0.0361)$ and $(4,0.0467)$ on the $(n, t)$-plane by

$$
t=(106 n+43) / 10000 \text {. }
$$

We have the following proposition which is proved also in [20].

Proposition 5. When $3 \leqq n<4$, the cubıc polynomial of $t$ :

$$
\begin{aligned}
\frac{(2 n-1)\left(n^{2}-n-3\right)}{6}-t\left[\frac{40 n^{4}+552 n^{3}-1498 n^{2}+1152 n-153}{240}\right. & \\
& +\frac{\left(n^{2}-n+1\right)\left\{3 n(12 n-13) p_{n}+18 n^{2}-28 n-4\right\}}{108} \\
& -\frac{(n-2)(n-3)\left(n^{4}-162 n^{3}+5140 n^{2}-6138 n+1159\right)}{16000(n-1)}
\end{aligned}
$$




$$
\begin{aligned}
& \left.-\frac{n\left(20 n^{4}-1595 n^{3}+4173 n^{2}-37481 n+4083\right)}{15(41-n)^{2}}\right] \\
& -t^{2}\left[\frac{160 n^{5}-288 n^{4}+1028 n^{3}-2347 n^{2}+1523 n-234}{480}\right. \\
& -\quad-\frac{(4 n-5)\left(n^{2}-n+1\right) p_{n}\left(6 n p_{n}+18 n^{2}-28 n-4\right)}{216} \\
& -\frac{(n-2)(n-3)(4 n-3)\left(n^{4}-162 n^{3}+5140 n^{2}-6138 n+1159\right)}{32000(n-1)} \\
& \left.\quad+\frac{10 n(n-2)(n-3)(4 n-3)(61-n)}{3(41-n)^{2}}\right] \\
& -t^{3} \frac{3(n-2)(n-3)(4 n-1)\left(8 n^{2}-8 n+3\right)}{320}
\end{aligned}
$$

is positive for $0 \leqq t \leqq(106 n+43) / 10000$, where $p_{n}$ is given by (4.5).

§5. Proof of $V\left(x, X_{n}(x)\right)>0$ for $X_{n}^{-1}\left(b_{n}\right)<x<1$ with $3 \leqq n \leqq 5$.

When $3 \leqq n \leqq 4$, we have

$$
V\left(x, X_{n}(x)\right)>0 \quad \text { for } \quad X_{n}^{-1}\left(1+(n-1) \frac{106 n+43}{10000}\right) \leqq x<1
$$

by means of Proposition 1 and Proposition 5 . When $4 \leqq n<4.5$, we have

$$
V\left(x, X_{n}(x)\right)>0 \quad \text { for } \quad X_{n}^{-1}\left(1+(n-1) \frac{6(2 n+73)}{10000}\right) \leqq x<1
$$

by means of Proposition 2 and Proposition 4 . And, when $4.5 \leqq n<5$, we have

$$
V\left(x, X_{n}(x)\right)>0 \quad \text { for } \quad X_{n}^{-1}\left(1+(n-1) \frac{565-14 n}{10000}\right) \leqq x<1
$$

by means of Proposition 2 and Proposition 3 . In this section, we set

$$
Z= \begin{cases}1+(n-1) \frac{106 n+43}{10000} & \text { for } 3 \leqq n<4, \\ 1+(n-1) \frac{6(2 n+73)}{10000} & \text { for } 4 \leqq n<4.5, \\ 1+(n-1) \frac{565-14 n}{10000} & \text { for } 4.5 \leqq n<5\end{cases}
$$

and we see easily that $Z$ is increasing with respect to $n$ in the above three ranges of $n$.

Now, noticing (5.1) (5.3), it is sufficient to prove that 


$$
V\left(x, X_{n}(x)\right)>0 \quad \text { for } \quad X_{n}^{-1}\left(b_{n}\right)<x<X_{n}^{-1}(Z(n))
$$

for our purpose. We shall also depend on the method used in $\S 6, \S 7$ of (VII). First we cite Proposition 1 and Proposition 3 in (VII).

Proposition 6. When $n>2$, we have

$$
V\left(x, X_{n}(x)\right)>(n-1)^{n-1} K\left(n, X_{n}(x)\right) \quad \text { for } 0<x<1 \text {, }
$$

where

(5.7) $K(n, X):=\frac{w^{2} \sqrt{n-w}}{(1-w)^{5}}\left[\left\{-P_{2}(y)+\left(\frac{n-y}{n-1}\right)^{n-1} P_{3}(y)\right\}\right.$

$$
\begin{aligned}
& \left.\times\left\{\log \frac{n-y}{n-X}+\frac{n X y-(2 n-1) X+n-y}{(n-1) X(n-y)}\right\}+\frac{2 n(1-w)^{3}(1-X w)}{n-w}\right] \\
+ & \frac{3 y^{2}}{(1-y)^{3} \sqrt{n-y}}\left[2 n-1-w-\left(\frac{n-w}{n-1}\right)^{n-1}\left\{n-w+(n-1) w^{2}\right\}\right] \\
- & \frac{X}{(n-1)(X-1)^{3} \sqrt{n-X}}\left[Q_{2}(X)+w Q_{3}(X)\right],
\end{aligned}
$$

where $X=X_{n}(x)$,

$$
Q_{2}(z):=\left(4 n^{2}+2 n-3\right) z^{3}-\left(4 n^{3}+18 n^{2}-19 n+3\right) z+n(4 n-1)(4 n-3),
$$$$
\text { and } w=w(n, X), y=y(n, X) \text { by (1.16), (1.20). }
$$

Remark. In Proposition 6, we can replace $y$ by

$$
\tilde{y}=\tilde{y}(n, X ; C):=\left(\frac{n-X}{n-1}\right)^{n-k_{n}(C)} \text { for } 1<X \leqq C, \quad \text { with } 1<C \leqq \frac{5 n-1}{2(n+1)},
$$

by means of Lemma 1.4. With this replacement, we denote this function by $\widehat{K}(n, X ; C)$ and call the corresponding statement Proposition $6^{\prime}$ in the following.

Proposition 7. $K(n, X)$ satisfies the following inequality: Let $n \geqq 3$ and $1<X<b_{n}$, and $n_{1}, n_{2}, X_{1}, X_{2}$ be such that

then

$$
3 \leqq n_{1} \leqq n \leqq n_{2}, \quad a_{n} \leqq X_{1} \leqq X \leqq X_{2}<b_{n},
$$

$$
K(n, X) \geqq K\left(n_{1}, n_{2} ; X_{1}, X_{2}\right),
$$

where

$$
K\left(n_{1}, n_{2} ; X_{1}, X_{2}\right)=\frac{\left(w\left(n_{1}, X_{2}\right)\right)^{2} \sqrt{n_{1}-w\left(n_{1}, X_{2}\right)}}{\left(1-w\left(n_{1}, X_{2}\right)\right)^{5}}
$$




$$
\begin{aligned}
& \times\left[\left\{-P_{2}\left(n_{2}, y\left(n_{2}, X_{1}\right)\right)+\left(\frac{n_{1}-y\left(n_{2}, X_{1}\right)}{n_{1}-1}\right)^{n_{1}-1} P_{3}\left(n_{1}, y\left(n_{2}, X_{1}\right)\right)\right\}\right. \\
& \quad \times\left\{\log \frac{n_{2}-y\left(n_{2}, X_{1}\right)}{n_{2}-X_{1}}+\frac{n_{2} X_{1} y\left(n_{2}, X_{1}\right)-\left(2 n_{2}-1\right) X_{1}+n_{2}-y\left(n_{2}, X_{1}\right)}{\left(n_{2}-1\right) X_{1}\left(n_{2}-y\left(n_{2}, X_{1}\right)\right)}\right\} \\
& \left.+\frac{2 n_{2}\left\{1-w\left(n_{2}, X_{2}\right)\right\}^{3}\left\{1-X_{1} w\left(n_{2}, X_{1}\right)\right\}}{n_{2}-w\left(n_{2}, X_{1}\right)}\right] \\
& +\frac{3\left(y\left(n_{2}, X_{1}\right)\right)^{2}}{\left(1-y\left(n_{2}, X_{1}\right)\right)^{3} \sqrt{n_{1}-y\left(n_{2}, X_{1}\right)}}\left[2 n_{2}-1-w\left(n_{1}, X_{2}\right)\right. \\
& \left.-\left(\frac{n_{2}-w\left(n_{1}, X_{2}\right)}{n_{2}-1}\right)^{n_{2}-1}\left\{n_{2}-w\left(n_{1}, X_{2}\right)+\left(n_{2}-1\right)\left(w\left(n_{1}, X_{2}\right)\right)^{2}\right\}\right] \\
& -\frac{X_{1} Q_{2}\left(n_{2}, X_{1}\right)}{\left(n_{1}-1\right)\left(X_{1}-1\right)^{3} \sqrt{n_{1}-X_{1}}}-\frac{X_{2} w\left(n_{1}, X_{2}\right) Q_{3}\left(n_{1}, X^{*}\right)}{\left(n_{2}-1\right)\left(X_{2}-1\right)^{3} \sqrt{n_{2}-X_{2}}}
\end{aligned}
$$

and $X^{*}=X_{1}$ for $n \geqq 4.46$, and $X^{*}=X_{2}$ when $X>\gamma_{n}$ and $X^{*}=X_{1}$ when $X \leqq \gamma_{n}$ for $(8+\sqrt{34}) / 4(=3.4577) \leqq n<4.46$, and $X^{*}=X_{2}$ for $3 \leqq n<(8+\sqrt{34}) / 4$, and $P_{2}(n, x)$, $P_{3}(n, x), Q_{2}(n, x), Q_{3}(n, x)$ stand for $P_{2}(x), P_{3}(x), Q_{2}(x), Q_{3}(x)$ with $n$ by (1.6), (1.7), (5.8), (5.9) respectively, and

$$
\gamma_{n}=\frac{2 n^{3}-9 n^{2}+13 n-3+\sqrt{4 n^{6}-12 n^{5}-23 n^{4}+66 n^{3}-11 n^{2}-24 n+9}}{3(n-1)(2 n-3)} .
$$

Remark. Regarding $w$ and $y$ in Proposition 7, we used the inequalities

$$
\begin{aligned}
& w\left(n_{1}, X_{2}\right) \leqq w(n, X) \leqq w\left(n_{2}, X_{1}\right), \\
& y\left(n_{1}, X_{2}\right) \leqq y(n, X) \leqq y\left(n_{2}, X_{1}\right)
\end{aligned}
$$

by Lemma 1.2 and Lemma 1.5. Corresponding to Proposoition 6', we consider $C$ as a decreasing function of $n$ satisfying

$$
C=C(n) \leqq \frac{5 n-1}{2(n+1)},
$$

then we obtain the inequalities:

$$
\left(\frac{n_{1}-X_{2}}{n_{1}-1}\right)^{n_{2}-k_{n_{2}}\left(C\left(n_{2}\right)\right)} \leqq\left(\frac{n-X}{n-1}\right)^{n-k_{n}(C(n))} \leqq\left(\frac{n_{2}-X_{1}}{n_{2}-1}\right)^{n_{1}-k_{n} n_{1}^{\left(C\left(n_{1}\right)\right)}}
$$

by Lemma 1.3. We denote the right hand side of (5.12) replaced $y\left(n_{2}, X_{1}\right)$ with

$$
\left(\frac{n_{2}-X_{1}}{n_{2}-X_{1}}\right)^{n_{1}-k_{n}\left(C\left(n_{1}\right)\right)}
$$

by the notation $\tilde{K}\left(n_{1}, n_{2} ; X_{1}, X_{2} ; C\right)$. Then, we obtain

$$
\tilde{K}(n, X ; C)>\tilde{K}\left(n_{1}, n_{2} ; X_{1}, X_{2} ; C\right)
$$




$$
\text { for } 3 \leqq n_{1} \leqq n \leqq n_{2} \text { and } a_{n} \leqq X_{1} \leqq X \leqq X_{2}<\min \left(b_{n}, C(n)\right) \text {. }
$$

We also call the corresponding statement Proposition $7^{\prime}$.

We computed approximately the values of $Y=Y(n)$ such that $K(n, Y)=0$ with $1<Y<n$ for each $n(5 \leqq n \leqq 9.7)$ with step $1 / 100$ and made Table 1 and Table $1^{\prime}$ of (VII) for the values of $n$ with step $1 / 10$. We made analogously Table 1 for $3 \leqq n \leqq 5$.

Table 1.

\begin{tabular}{|l|c|c|c||c|c|c|c|}
\hline$n$ & $Y$ & $Z$ & $C_{1}$ & $n$ & $Y$ & $Z$ & $C_{1}$ \\
\hline 3.0 & 1.4445 & 1.0722 & 1.45 & 4.0 & 1.3789 & 1.1458 & 1.38 \\
3.1 & 1.4318 & 1.0780 & 1.443 & 4.1 & 1.3754 & 1.1510 & 1.378 \\
3.2 & 1.4218 & 1.0840 & 1.436 & 4.2 & 1.3721 & 1.1562 & 1.376 \\
3.3 & 1.4136 & 1.0903 & 1.429 & 4.3 & 1.3689 & 1.1615 & 1.374 \\
3.4 & 1.4068 & 1.0968 & 1.422 & 4.4 & 1.3659 & 1.1668 & 1.372 \\
3.5 & 1.4008 & 1.1035 & 1.415 & 4.5 & 1.3629 & 1.1722 & 1.37 \\
3.6 & 1.3956 & 1.1103 & 1.408 & 4.5 & 1.3629 & 1.1757 & 1.37 \\
3.7 & 1.3909 & 1.1175 & 1.401 & 4.6 & 1.3600 & 1.1802 & 1.368 \\
3.8 & 1.3866 & 1.1248 & 1.394 & 4.7 & 1.3572 & 1.1847 & 1.366 \\
3.9 & 1.3826 & 1.1323 & 1.387 & 4.8 & 1.3545 & 1.1891 & 1.364 \\
4.0 & 1.3789 & 1.1401 & 1.38 & 4.9 & 1.3518 & 1.1935 & 1.362 \\
\hline
\end{tabular}

Observing this table and noticing that $(5 n-1) / 2(n+1) \geqq 7 / 4=1.75$ for $n \geqq 3$, we can prove

$$
Y=Y(n)<\frac{5 n-1}{2(n+1)} .
$$

This inequality is implied from

$$
K\left(n, \frac{5 n-1}{2(n+1)}\right)>0,
$$

which is proved by showing $K\left(n_{1}, n_{2} ; X_{1}, X_{2}\right)>0$ for suitably selected $n_{1}, n_{2}, X_{1}$, $X_{2}$ as

$$
3 \leqq n_{1}<n_{2} \leqq 5 \quad \text { and } \quad X_{1} \leqq \frac{5 n_{1}-1}{2\left(n_{1}+1\right)}<\frac{5 n_{2}-1}{2\left(n_{2}+1\right)} \leqq X_{2},
$$

by means of Proposition 6 and Proposition 7 . Therefore, we can use Proposition $6^{\prime}$ and Proposition $7^{\prime}$ with suitably selected decreasing function $C=C(n)$ of $n$ in the following arguments.

First, setting 


$$
C=C_{1}(n)= \begin{cases}(166-7 n) / 100 & \text { for } 3 \leqq n<4 \\ (73-n) / 50 & \text { for } 4 \leqq n \leqq 5,\end{cases}
$$

we can prove $Y(n)<C_{1}(n)$, by showing $K\left(n, C_{1}(n)\right)>0$ which is assured by the same way as described above. Then, we set

$$
K_{1}(n, X):=\tilde{K}\left(n, X ; C_{1}(n)\right)
$$

and let $Y_{1}(n)$ be the solution of the equation

Setting

$$
K_{1}\left(n, Y_{1}\right)=0 \text { with } 1<Y_{1}<n \text { for } 3 \leqq n \leqq 5 .
$$

$$
C=C_{2}(n)= \begin{cases}(175-11 n) / 100 & \text { for } 3 \leqq n<4 \\ (37-n) / 25 & \text { for } 4 \leqq n \leqq 5 .\end{cases}
$$

We can prove $Y_{1}(n)<C_{2}(n)$, by showing $K_{1}\left(n, C_{2}(n)\right)>0$, which can be assured by the same way above, using $K_{1}(n, X)$ and $K_{1}\left(n_{1}, n_{2} ; X_{1}, X_{2}\right)$ constructed suitably from $\tilde{K}\left(n_{1}, n_{2} ; X_{1}, X_{2} ; C\right)$ in place of $K(n, X)$ and $K\left(n_{1}, n_{2} ; X_{1}, X_{2}\right)$.

By the same way, for $j \geqq 1$ we suppose inductively $Y_{j}(n)$ is defined by

$$
K_{j}\left(n, Y_{\jmath}(n)\right)=0 \quad \text { with } 1<Y_{j}(n)<n \text { for } 3 \leqq n \leqq 5
$$

and a decreasing function $C=C_{j+1}(n)$ such that

$$
Y_{j}(n)<C_{\jmath+1}(n) \leqq Y_{j}(n)+\frac{1}{10^{4}},
$$

and then we put

$$
K_{\jmath+1}(n, X):=\tilde{K}\left(n, X ; C_{\jmath+1}(n)\right) .
$$

By this principle of making $K_{j}(n, X), Y_{j}(n), C_{j}(n)$ and $K_{j}\left(n_{1}, n_{2} ; X_{1}, X_{2}\right)$, we obtained $C_{j}(n)$ for $3 \leqq j \leqq 7$ as follows :

$$
\begin{aligned}
& C_{3}(n)= \begin{cases}(1804-133 n) / 1000 & \text { for } 3 \leqq n<4 \\
(76-3 n) / 50 & \text { for } 4 \leqq n<5,\end{cases} \\
& C_{4}(n)= \begin{cases}(1848-149 n) / 1000 & \text { for } 3 \leqq n<4 \\
(189-8 n) / 125 & \text { for } 4 \leqq n \leqq 5,\end{cases} \\
& C_{5}(n)= \begin{cases}(1873-158 n) / 1000 & \text { for } 3 \leqq n<4 \\
\left(8 n^{2}-152 n+1723\right) / 1000 & \text { for } 4 \leqq n \leqq 5,\end{cases} \\
& C_{6}(n)= \begin{cases}(18923-1647 n) / 10000 & \text { for } 3 \leqq n<4 \\
\left(80 n^{2}-1428 n+14065\right) / 7800 & \text { for } 4 \leqq n \leqq 5,\end{cases} \\
& C_{7}(n)=(16634-1084 n) / 110000 \text { for } 4 \leqq n \leqq 4.5,
\end{aligned}
$$


where we computed the values of $Y_{j}(n)$ for $n$ with step $1 / 100$.

Here, we cite the approximately computed values of $Y_{j}(n)$ for the values of $n$ with step $1 / 10$ as in Table 2.

Table 2 .

\begin{tabular}{|l|c|c|c|c|c|c|c|}
\hline$n$ & $Z$ & $Y_{1}$ & $Y_{2}$ & $Y_{3}$ & $Y_{4}$ & $Y_{5}$ & $Y_{6}$ \\
\hline 3.0 & 1.0722 & 1.4123 & 1.4044 & 1.4001 & 1.3989 & 1.3982 & 1.3980 \\
3.1 & 1.0780 & 1.3950 & 1.3854 & 1.3799 & 1.3781 & 1.3771 & 1.3766 \\
3.2 & 1.0840 & 1.3803 & 1.3689 & 1.3622 & 1.3596 & 1.3582 & 1.3574 \\
3.3 & 1.0903 & 1.3675 & 1.3541 & 1.3462 & 1.3428 & 1.3409 & 1.3398 \\
3.4 & 1.0968 & 1.3560 & 1.3406 & 1.3312 & 1.3269 & 1.3245 & 1.3230 \\
3.5 & 1.1035 & 1.3455 & 1.3279 & 1.3171 & 1.3118 & 1.3088 & 1.3069 \\
3.6 & 1.1103 & 1.3357 & 1.3159 & 1.3035 & 1.2971 & 1.2935 & 1.2911 \\
3.7 & 1.1175 & 1.3265 & 1.3043 & 1.2902 & 1.2827 & 1.2784 & 1.2755 \\
3.8 & 1.1248 & 1.3177 & 1.2931 & 1.2772 & 1.2685 & 1.2634 & 1.2600 \\
3.9 & 1.1323 & 1.3093 & 1.2821 & 1.2644 & 1.2543 & 1.2485 & 1.2444 \\
4.0 & 1.1401 & 1.3012 & 1.2713 & 1.2516 & 1.2402 & 1.2335 & 1.2288 \\
\hline 4.0 & 1.1458 & 1.3012 & 1.2760 & 1.2560 & 1.2425 & 1.2347 & 1.2298 \\
4.1 & 1.1510 & 1.2592 & 1.2689 & 1.2476 & 1.2336 & 1.2242 & 1.2183 \\
4.2 & 1.1562 & 1.2895 & 1.2621 & 1.2394 & 1.2250 & 1.2141 & 1.2072 \\
4.3 & 1.1615 & 1.2841 & 1.2555 & 1.2314 & 1.2166 & 1.2042 & 1.1964 \\
4.4 & 1.1668 & 1.2788 & 1.2491 & 1.2237 & 1.2084 & 1.1946 & 1.1858 \\
4.5 & 1.1757 & 1.2738 & 1.2429 & 1.2161 & 1.2004 & 1.1853 & 1.1756 \\
4.6 & 1.1802 & 1.2689 & 1.2369 & 1.2087 & 1.1926 & 1.1763 & 1.1657 \\
4.7 & 1.1847 & 1.2641 & 1.2310 & 1.2015 & 1.1849 & 1.1675 & 1.1560 \\
4.8 & 1.1891 & 1.2595 & 1.2253 & 1.1944 & 1.1774 & 1.1590 & 1.1466 \\
4.9 & 1.1935 & 1.2551 & 1.2198 & 1.1875 & 1.1700 & 1.1507 & 1.1376 \\
5.0 & 1.1980 & 1.2507 & 1.2143 & 1.1806 & 1.1628 & 1.1427 & 1.1280 \\
\hline
\end{tabular}

Looking over Table 2, we find that $Y_{6}(n)<Z(n)$ for the values of $n(4.5 \leqq n \leqq 5)$, which is derived from

$$
K_{6}(n, Z(n))>0, \quad \text { where } Z(n)=1+\frac{(n-1)(565-14 n)}{10000} .
$$

This inequality can be proved by showing

$$
K_{6}\left(n_{1}, n_{2} ; X_{1}, X_{2}\right)>0
$$

for suitably selected $n_{1}, n_{2}, X_{1}, X_{2}$ as

$$
4.5 \leqq n_{1}<n_{2} \leqq 5 \text { and } X_{1} \leqq Z\left(n_{1}\right)<Z\left(n_{2}\right) \leqq X_{2} \text {, }
$$

by the same reason as before. 
By means of Proposition $6^{\prime}$ and Propoaition $7^{\prime}$ and the above results for $4.5 \leqq n \leqq 5$, we obtain

$$
V\left(x, X_{n}(x)\right)>0 \quad \text { for } 0<x<1,
$$

which implies Theorem $\mathrm{C}$ for $4.5 \leqq n \leqq 5$. Hence, we have obtained the main theoarem of this paper as follows.

THEOREM C. The period function $T$ as a function of $\tau$ and $n$ is monotone decreasing with respect to $n \geqq 4.5$ for any fixed $\tau(0<\tau<1)$.

Remark. The author has expected to prove the above Theorem $\mathrm{C}$ foar $3 \leqq$ $n \leqq 5$ in the early stage of this work. But, when he proceeded the same arguments as above for $3 \leqq n \leqq 4.5$, he found that it takes too long steps to obtain the same result for this interval of $n$, especially as $n$ comes near 3 , by means of the micro computor used up to the present. He will try it by improving Proposition 1 and Proposition 2 and using a more elaborate machine to the interval $3 \leqq n \leqq 4.5$. We know that the methods used until now does not work well for the proof of Conjecture C for $2 \leqq n \leqq 3$ by Lemma 8.1 of (III). Therefore, for this interval we have to develop new methods, which may be applicable to the interval $3 \leqq n \leqq 4.5$.

\section{REFERENCES}

[1] S.S. Chern, M. Do CARmo And S. Kobayashi, Minimal Submanifolds of a sphere with second fundamental form of constant length, Functional Analysis and Related Fields, Springer-Verlag, 1970, 60-75.

[2] S. FuruYA, On periods of periodic solutions of a certain nonlinear differential equation, Japan-United States Seminar on Ordinary Differential and Functional Equations, Lecture Notes in Mathematics, Springer-Verlag, 243 (1971), 320-323.

[3] W. Y. Hsiang and H. B. LAwson, JR., Minimal submanifolds of low cohomogeneity, J. Diff. Geometry, 5 (1970), 1-38.

[4] T. Otsuki, Minimal hypersurfaces in a Riemannian manifold of constant curvature, Amer. J. Math., 92 (1970), 145-173.

[5] T. OTsuki, On integral inequalities related with a certain nonlinear differential equation, Proc. Japan Acad., 48 (1972), 9-12.

[6] T. Otsuki, On a 2-dimensional Riemannian manifold, Differential Geometry, in honor of K. Yano, Kinokuniya, Tokyo, 1972, 401-414.

[7] T. OTsuki, On a family of Riemannian manifolds defined on an m-disk, Math. J. Okayama Univ., 16 (1973), 85-97.

[8] T. OTsuki, On a bound for periods of solutions of a certain nonlinear differential equation (I), J. Math. Soc. Japan, 26 (1974), 206-233.

[9] T. Otsuki, On a bound for periods of solutions of a certain nonlinear differential equation (II), Funkcialaj Ekvacioj. 17 (1974), 193-205.

[10] T. OTsuki, Geodesics of $O_{n}^{2}$ and an analysis on a related Riemann surface, Tôhoku Math. J., 28 (1976), 411-427.

[11] T. OTsuki, A certain property of geodesics of the family of Riemannian mani- 
folds $O_{n}^{2}$ ( I ), Proc. of Japan-United States Seminar on Minimal Submanifolds, including Geodesics, Kaigai, Tokyo, 1978, 173-192.

[12] T. OTsuki, A certain property of geodesics of the family of Riemannian manifolds $O_{n}^{2}$ (II), Kodai Math. J., 2 (1979), 211-242.

[13] T. OTsukI, A certain property of geodesics of the family of Riemannian manifolds $O_{n}^{2}$ (III), Kodai Math. J., 4 (1981), 28-70.

[14] T. OTsuki, A certain property of geodesics of the family of Riemannian manifolds $O_{\pi}^{2}$ (IV), Kodai Math. J., 5 (1982), 160-199.

[15] T. OTsuki, A certain property of geodesics of the family of Riemannian manifolds $O_{n}^{2}$ (V), Kodai Math. J., 5 (1982), 454-481.

[16] T. OTsuki, A certain property of geodesics of the family of Riemannian manifolds $O_{n}^{2}$ (VI), Kodai Math. J., 7 (1984), 326-364.

[17] T. OTsuki, Certain inequalities related with a non-linear differential equation (I), (II), TRU Math., 20 (1984), 23-68, 69-89.

[18] T. OTsukI, A certain property of geodesics of the family of Riemannian manifolds $O_{n}^{2}$ (VI), Kodai Math. J., 8 (1985) , 375-419.

[19] T. OTsuki, Certain inequalities and constants related with a nonlinear differential equation, TRU Math., 21 (1985), 5-35.

[20] T. OTsukI, Certain inequalities and constants related with a nonlinear differential equation (II), to TRU Math., 21 (1985), 241-268.

[21] T. OTsuki, A family of 2-dimensional Riemannian manifolds joining the noneuclidean planes, TRU Math., 17 (1981), 129-139.

[22] M. Maeda And T. Otsuki, Models of the Riemannian manifolds $O_{n}^{2}$ in the Lorontzian 4-space, J. Diff. Geometry, 9 (1974), 97-108.

[23] M. URABE, Computations of periods of a certain nonlinear autonomous oscilations, Study of algorithms of numerical computations, Sūrikaiseki Kenkyūsho Kōkyū-roku, 149 (1972), 111-129 (Japanese).

Department of Mathematics

SCIENCE UNIVERSity OF TOKYO

W AKAMIYA-CHO, 26, SHINJUKU-KU

TOKYO, JAPAN 162 